\begin{tabular}{|c|c|c|c|}
\hline Eiszeitalter u. Gegenwart & $\mathbf{4 5}$ & $\begin{array}{c}109-129 \\
10 \mathrm{Abb} .\end{array}$ & Hannover 1995 \\
\hline
\end{tabular}

\title{
Eruptionsgeschichte und Deckschichtenfolge der Wannenköpfe-Vulkangruppe in der Osteifel
}

\author{
MANFRED FreChEN*) \\ Vulcanic sequences, loess-paleosol sequences, interglacial soils, tephrochronological investigations, \\ stratigraphy, East-Eifel
}

\begin{abstract}
Kurzfassung: Die Wannenköpfe gehören zu einer Gruppe von Schlackenkegeln, die unter interstadialen Verhältnissen zu Beginn der vorletzten Kaltzeit im Osteifel-Vulkanfeld ausbrachen. Durch den intensiven Lavaabbau sind derzeit imposante Einblicke in den vulkanologischen Formenschatz und die verschiedenen Eruptionsphasen eines Schlackenkegels gegeben. Die pyroklastische Abfolge beginnt mit phreatomagmatischen Ablagerungen eines initialen Maares. In einer zweiten nichtphreatomagmatischen Phase kommt es zur strombolianischen Förderung von Schlacken und damit zum Aufbau des Schlackenkegels. Im Bereich der Wannen-Vulkangruppe sind Lavaströme sowohl im Westen als auch im Osten ausgeflossen. Aus der Finalphase stammen die Basalt- und Tuffgänge. Mindestens einer dieser Tuffgänge erreichte die Krateroberfläche. Dabei wurde wenig nebengesteinsreiches, feinkörniges Material gefördert. Dieses Material wurde hydrothermal stark zersetzt und liegt als toniges Sediment an der Basis der Kratermulde vor (Profil B).
\end{abstract}

In den Kratermulden und den Depressionen zwischen einzelnen Schlackenkegeln bildeten sich Sedimentfallen, in denen besonders für den älteren Teil der vorletzten Kaltzeit eine mehr oder weniger lückenlose Sedimentabfolge vorhanden ist. Die Deckschichten beginnen mit Aschen- und Lapilli-Fallablagerungen sowie distalen phreatomagmatischen Ablagerungen benachbarter Eruptionszentren. Die Asche- und Lapillischichten können zum Teil mit den Tephren anderer Kraterprofile korreliert werden.

Zum Hangenden hin sind Horizonte mit Humusanreicherungen zwischengeschaltet, die als Äquivalente von interstadialen, frühsaalezeitlichen Böden interpretiert werden. Die Deckschichtenfolge wird durch einen rotbraunen BtRest einer Parabraunerde, der mit dem Eem-Boden korreliert, untergliedert. Unterweichselzeitliche Humuszonen, humose Fließerden und Lehmbröckelsande lagern diskordant auf dem interglazialen Boden. Die Profile schließen mit einer interstadialen Bodenbildung ab, dem Alleröd-Boden, der sich auf ungegliedertem oberweichselzeitlichem Löß gebildet hat. Darüber lagert der Laacher See-Bims.

Aufgrund der tephrochronologischen und lößstratigraphischen Untersuchungen ist eine Kombination der Löß/Paläobodenabfolge der Wannenköpfe mit denen des Tönchesberges und anderer Schlackenkegelkomplexe

\footnotetext{
*) Anschrift des Verfassers: Dr. M. Frechen, Abt. Quartärgeologie, Geologisches Institut, Universität zu Köln, Zülpicher Str. 49, $50674 \mathrm{Köln}$
}

möglich. Aus der Kombination der Profile ist eine genaue Einstufung des Ausbruches der Osteifel-Schlackenkegelkomplexe sowie eine Rekonstruktion der paläoklimatischen und paläoökologischen Entwicklung der Osteifel für die letzten 200.000 Jahre ableitbar. Die Wannenköpfe sind unter interstadialen, waldsteppenartigen Klimaverhältnissen ausgebrochen. Nur unwesentlich später sind der Tönchesberg, die Eiterköpfe, sowie der Korretsberg und der Plaidter Hummerich unter ähnlichen klimatischen Bedingungen ausgebrochen. Für den Ausbruch der HüttenbergTephra zu Beginn des vorletzten Interglazials gibt es an den Wannenköpfen keine Belege.

\section{[Eruption sequence and sediment sequence on top of the Wannenköpfe scoria complex]}

Abstract: The Wannenköpfe scoria complex of the East-Eifel Volcanic Field in Germany has preserved a remarkable record of glacial and interglacial sediments in inter- and intracrater depressions. Due to intensive mining, the different eruption cycles of typical scoria cones are visible in the quarry walls. The pyroclastic formation starts with phreatomagmatic deposits of an initial maar. In a nonphreatomagmatic phase scoria is erupted by strombolian activity. Lava flows are found around the scoria cones. In the final phase basaltic magma and fluidized sediment intrude into the volcano.

In the past the volcanic craters have acted as sediment traps. The loess/paleosol sequences provide a more or less continuous record of climatic changes especially for the early penultimate glaciation.

The volcanic sequence starts with "fall out" and flow deposits from neighbouring eruption centers of the Wannen scoria group. Above these deposits lie intercalated horizons, rich in humic material.

Higher in the sequence loess layers and their derivatives, subdivided by a thick redbrownish Bt horizon of an interglacial soil ("Parabraunerde"), occur within the inter- and intracrater depressions. On the top of the interglacial soil (stage 5e) steppe soils, humic reworked sediments and pellet sands from the early part of the last glaciation (stage $5 \mathrm{~d}-\mathrm{a})$ are well preserved and reflect the climatic and environmental development after the last interglacial optimum. Some sediments from the upper part of the last glaciation have been eroded out. Comparison and subsequent combination of different crater sections from scoria cones such as Eiterköpfe and Tönchensberg have been made using te- 
phrochronological and stratigraphic investigations. A detailed chronological reconstruction of the East-Eifel Volcanic Field is achieved.

The Wannenköpfe scoria cone erupted under interstadial climatic conditions. The scoria cones of Tönchesberg, Eiterköpfe, Korretsberg and Plaidter Hummerich erupted shortly after the Wannenköpfe complex under similar climatic and environmental conditions. There is no evidence that the Hüttenberg tephra erupted at the beginning of the penultimate interglaciation.

\section{Inhaltsverzeichnis}

1 Einleitung

2 (Paläo-)Geomorphologie

3 Eruptionsgeschichte der Wannenköpfe
3.1 Phreatomagmatische Initialphase und Maarbildung

3.2 Strombolianische Schlackenwurftätigkeit

3.3 Finalphase mit Intrusionen von Tuff- oder Basaltgängen

3.4 Effusive Phase

3.5 Tephra-Ablagerungen benachbarter Eruptionszentren

4 Sedimentologisch-pedologische Ergebnisse/Profilbeschreibungen

4.1 Profil A

4.2 Profil B

4.3 Profil D („Canyonprofil“)

5 Diskussion

6 Dank

7 Schriftenverzeichnis

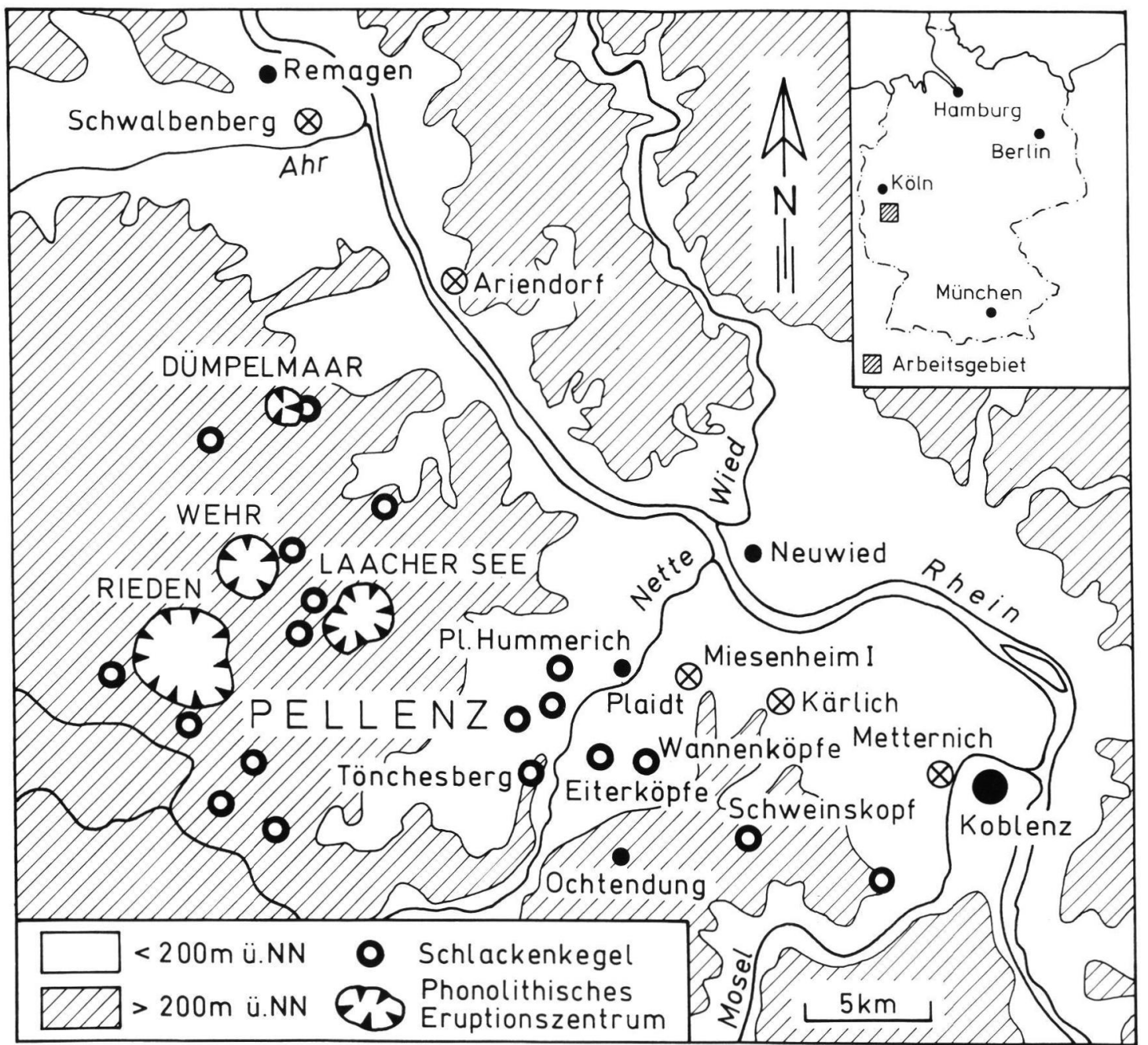

Abb. 1: Lage des Wannenköpfe-Schlackenkegelkomplexes in der Osteifel. Kraterprofile sind als schwarze offene Kreise $(\mathbf{o})$ und Umlandprofile als offene Kreise mit Kreuz $(\otimes)$ gekennzeichnet.

Fig. 1: Map showing the location of the Wannenköpfe scoria complex in the East Eifel Volcanic Field, Germany. Crater sections are marked by an open black circle (o), other loess sections by an open circle with a cross $(\Theta)$. 


\section{Einleitung}

Die Wannenköpfe gehören zu den rund 100 kleineren alkalibasaltischen Schlackenkegeln der Osteifel, die während des Mittel- und Oberpleistozäns ausbrachen. Durch den intensiven Lavaabbau seit den 1970er Jahren bestehen hervorragende Aufschlußverhältnisse, in denen der Aufbau und die verschiedenen Eruptionsphasen von Schlackenkegeln bzw. Schlackenkegelkomplexen des Osteifel-Vulkanfeldes untersucht werden können (SCHMINCKE 1977).

Die Wannenköpfe befinden sich auf der durch zahlreiche Mikroerdbeben immer noch aktiven Nordwest-Südost ausgerichteten Ochtendunger Störungszone (AHORNER 1983: 210). Auf dieser tektonischen Linie liegen zwischen dem SchweinskopfKarmelenberg und Birkenkopf im Südosten und dem Bausenberg im Nordwesten zahlreiche weitere, für die zeitliche Stellung des Osteifel-Vulkanismus wichtige Schlackenkegelkomplexe, u. a. die Eiterköpfe, der Tönchesberg, der Plaidter Hummerich und der Korretsberg (Abb. 1).

Im Bereich der Wannenköpfe sind derzeit verschiedene Maar-Ablagerungen und Schlackenkegel in mehreren Steinbrüchen aufgeschlossen. Anhand der abbaubedingt wechselnden Steinbruchwände kann die Eruptionsgeschichte des WannenköpfeSchlackenkegelkomplexes detailliert rekonstruiert werden.

Die Kratermulden und die Depressionen zwischen den einzelnen Schlackenkegeln bildeten über geologisch lange Zeiträume Sedimentfallen. Durch eine Verknüpfung und Kombination der etwa zeitgleichen Abfolgen wird eine Rekonstruktion der paläoklimatischen und paläoökologischen Bedingungen der letzten beiden Glazial-/Interglazialzyklen für die Osteifel ermöglicht. Für die Decksedimente des Tönchesberges, durch die eine mehr oder weniger lückenlose Abfolge saale- und unterweichselzeitlicher Sedimente überliefert ist, liegen bereits umfangreiche interdisziplinäre Untersuchungen vor (Boenigk \& Frechen im Druck). Des weiteren gibt es sedimentologisch-pedologische Untersuchungen der Decksedimente des Plaidter Hummerichs (SEMMEL 1991) und des Schweinskopf-Karmelenberges (FRECHEN in Vorbereitung).

Mit Hilfe der Decksedimente und der Sedimente unterhalb des Schlackenkegelkomplexes erfolgt eine lößstratigraphische Einstufung und damit eine relative Datierung des Ausbruchs der Wannenköpfe. Die zwischengeschalteten Tephren erlauben Korrelationen mit weiteren mehr oder weniger zeitgleichen Schlackenkegeln des Osteifel-Vulkanfeldes.

In dieser Arbeit wird eine Bestandsaufnahme des vulkanologischen Formenschatzes und die Rekonstruktion des Eruptionsablaufs der Wannenköpfe vorgestellt, sowie die sedimentologisch-pedologischen und stratigraphischen Ergebnisse bezüglich der Deck- und Liegendsedimente. Die Profile A, B und $\mathrm{D}$ können aufgrund der petrographisch und geochemisch unterscheidbaren Tephralagen zu einem Gesamtprofil kombiniert werden (Abb. 9), das darüber hinaus mit dem Tönchesberg-Profil verknüpft werden kann (Abb. 10). Der Begriff Saale wird in dieser Arbeit für das vorletzte Glazial (Sauerstoffisotopenstadien 7b bis 6), der Begriff Eem für das letzte Interglazial (Sauerstoffisotopenstadium 5e) und Weichsel für das letzte Glazial (Sauerstoffisotopenstadien $5 \mathrm{~d}$ bis 2) verwendet.

Eine kurze Beschreibung der vulkanischen Ablagerungen und eine tephrostratigraphische Korrelation mit den Abfolgen der Schlackenkegelkomplexe Tönchesberg, Plaidter Hummerich und Korretsberg erfolgte bereits durch BOGAARD \& SCHMINCKE (1990: 178 ff) und SCHMINCKe, BOGAARD \& FREUndT (1990: $105 \mathrm{ff}$ ). Erste Ergebnisse der ur- und frühgeschichtlichen Funde finden sich bei Justus, Urmersbach \& URMERSBACH (1987), die Untersuchungsergebnisse bezüglich der Mollusken und Kleinsäuger aus der ur- und frühgeschichtlichen Grabung bei KolfsCHOTEN \& ROTH (im Druck).

\section{2 (Paläo-)Geomorphologie}

Der Schlackenkegelkomplex der Wannenköpfe ist Teil der Wannenvulkangruppe, einer spätmittelpleistozänen Vulkangruppe des Osteifel-Vulkanfeldes. Die Wannenköpfe liegen zwischen den Ortschaften Ochtendung, Plaidt und Saffig (Abb. 1 und 2) östlich der Straße Ochtendung/Saffig (Topographische Karte 1:25.000, Blatt 5610 Bassenheim). Die östliche Wannenvulkangruppe besteht aus den Schlackenkegelkomplexen der „Wannenköpfe“ und „In den Wannen" sowie dem Wannenfeld östlich der Straße Ochtendung/Saffig. Die westliche Wannengruppe besteht aus den „Eiterköpfen“, dem „Langenberg“, dem „Michelberg“ und einigen weiter nördlich gelegenen kleineren Vulkankuppen (Abb. 2).

Die beiden höchsten Erhebungen sind der Michelberg mit 276,60 m ü. NN und ein Punkt südöstlich der Wannenköpfe mit 287,8 m ü. NN, der allerdings durch den Lavaabbau abgetragen worden ist. Die Schlackenkegel haben eine Höhe von maximal 50 bis $80 \mathrm{~m}$ über der nicht vulkanischen Unterlage. Nach Quiring (1936: 35) ist der höchste Schlackenkegel der Michelberg mit einer Höhe von 110 m oberhalb der jüngeren Mittelterrasse. Die Morphologie der Schlackenkegelkomplexe vor dem intensiven Lavaabbau ist bei Von Dechen (1864: 396ff) beschrieben. Eine Rekonstruktion der ehemaligen Morphologie vor dem intensiven Lavaabbau wurde anhand von alten Karten und Luftbildern durchgeführt (Abb. 2). 


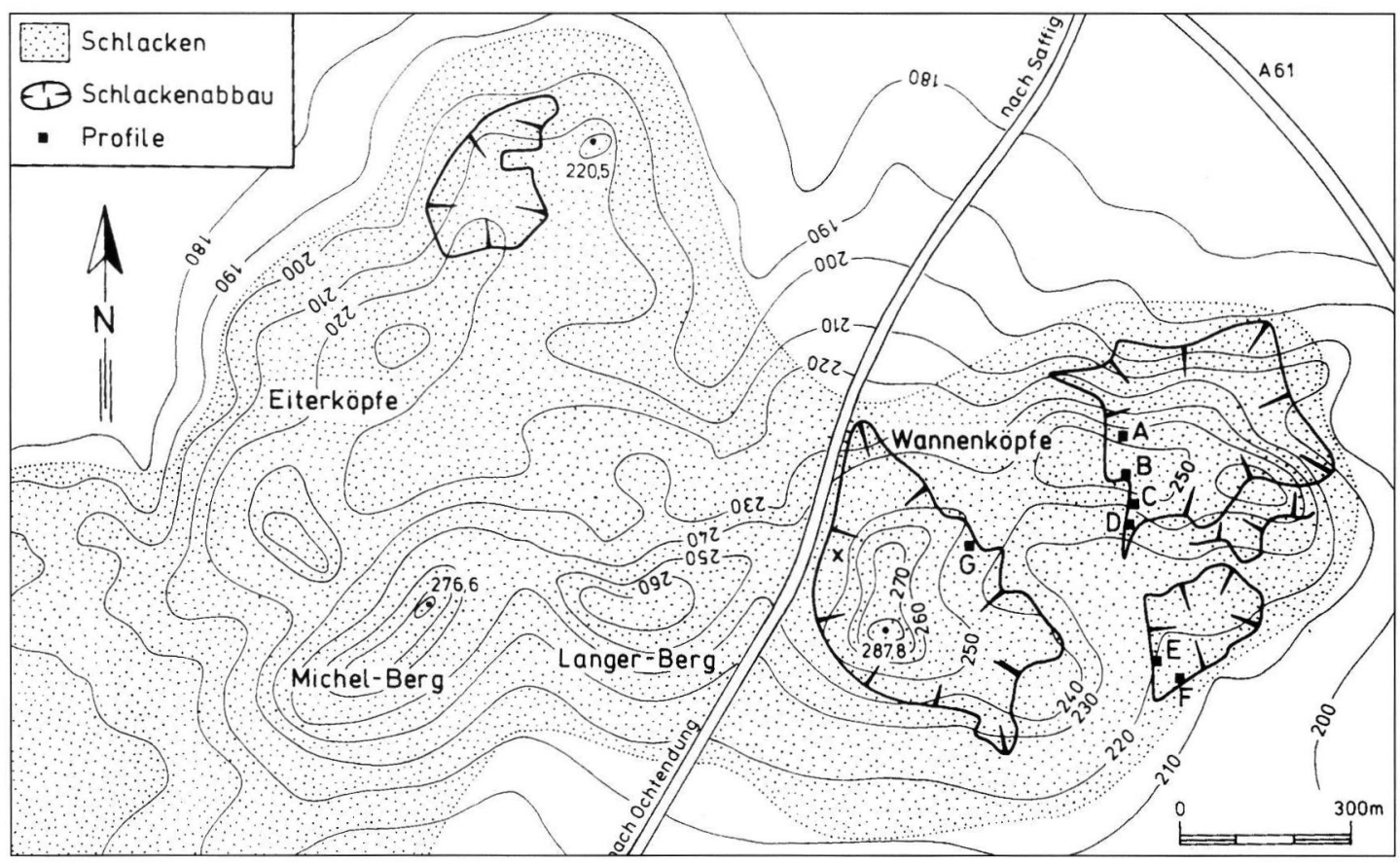

Abb. 2: Topographie der Wannenköpfe vor dem intensiven Lavaabbau. Lage der Profile, Abbaustand und Verbreitung der Schlacke.

Fig. 2: Topography of the Wannenköpfe scoria complex before intensive mining. Position of profiles and distribution of scoria in the field.

Die Ausdehnung des Schlackenkegelkomplexes ohne die Lavaströme beträgt etwa $2,7 \mathrm{~km}$ in Ost-/WestRichtung, in Nord-Süd-Richtung dagegen 1,3 km für die westliche und $0,8 \mathrm{~km}$ für die östliche Wannenvulkangruppe.

Die erste detaillierte Beschreibung der WannenSchlackenkegelkomplexe findet sich bei AHrENS (1932: 858), der zudem versuchte, die Lavavorkommen mit geomagnetischen Messungen abzugrenzen. Die Wannenvulkangruppe besteht nach AHrens (1932: 858) aus mindestens 12-15 selbständigen Eruptionszentren. Aufgrund der durch den intensiven Lavaabbau wesentlich verbesserten Aufschlußverhältnisse kommt man jedoch östlich der Straße von Ochtendung nach Saffig bereits auf etwa 15 -18 Eruptionszentren.

Der Wannenvulkankomplex wird im Westen und Osten von Lavaströmen umgeben. Im Westen erstrecken sich die Lavaströme bis zum Nettetal, in dem hervorragende Aufschlüsse bestehen. Östlich von Plaidt befindet sich der Lavastrom auf der nordwestlichen Uferseite der Nette. Mehrere übereinander geflossene Lavaströme sind in einem aufgelassenen kleinen Basaltsteinbruch südlich der Heseler Mühle aufgeschlossen. Die maximale Ausdehnung des westlichen Lavastromes, der bei Miesenheim immer noch eine Breite von $300 \mathrm{~m}$ hat, beträgt etwa 5,5 $\mathrm{km}$ in SW-NE-Richtung.
Der Lavastrom östlich der Wannenköpfe ist zweigeteilt. Der nördliche Lavastrom floß zunächst nach Osten ab, um dann der Morphologie folgend nach Nordwesten in Richtung Saffig weiter zu fließen. Die Länge dieses Lavastromes erreicht mindestens 1,3 $\mathrm{km}$. Der südliche Lavastrom mit einer Länge von mindestens $1,2 \mathrm{~km}$ floß ebenfalls zunächst nach Osten $\mathrm{ab}$, drehte dann nach Südosten und floß zuletzt nach Südwesten ab. Die Mächtigkeit der Lavaströme erreicht örtlich mehr als 20 m (QuiRING 1936: 35) bzw. 15 -18 m südlich von Plaidt (Ahrens 1932: 862).

Die Lavaströme, die nach Westen abflossen, liegen nach AHrens (1932: 861) zunächst einer älteren Mittelterrasse der Nette auf, die etwa 150-160 m ü. NN und damit $40 \mathrm{~m}$ über dem heutigen Flußbett der Nette liegt. Im Nettetal liegt ein Lavastrom der jüngeren Mittelterrasse auf (AHREns 1932: 865).

AHRENs berichtet von Resten basaltischer Lava westlich der Nette gegenüber von Burg Wernerseck. Die Unterkante dieses Lavastromes liegt $1 \mathrm{~km}$ südlich von Plaidt in der Höhe des jetzigen Talbodens, teilweise sogar etwas darunter (100-105 m ü. NN). An der Rauschermühle steht die Lava an beiden Ufern sowie im Bach selbst an. Südlich von Plaidt liegt die Lava aufgrund einer WSW-ENE-Störung tiefer, wobei nördlich der Störung die Lava am stärksten absank (Ahrens 1932: 866): Die Lava liegt dort so tief, 
daß die Nette sie noch nicht vollständig erodieren konnte (bei 85-90 $\mathrm{m}$ ü. NN). AHRENS (1932: 866) geht von einer Sprunghöhe der Störung von 5-10 m aus.

Im Bereich der Wannenköpfe ist nördlich von Profil E (Abb. 2) ein gut gesäulter Lavastrom aufgeschlossen, der ein ehemaliges Tälchen ausfüllte.

Das Liegende des Schlackenkegelkomplexes is derzeit an den Wannenköpfen unterhalb der Maar-Ablagerungen aufgeschlossen (Profil A). Die Morphologie, basierend auf der Höhenlage der Basis der Maar-Ablagerungen, ist auf 50-60 m eben. Das Profil B befindet sich am Gipfel des Schlackenkegels in einer ehemaligen kleinen Kratermulde. Es handelte sich um eine Sedimentfalle, die aufgrund der geringen Tiefe und Ausdehnung des Kraters relativ schnell und früh durch pyroklastische Ablagerungen und äolischen Sedimenteintrag gefüllt worden ist.

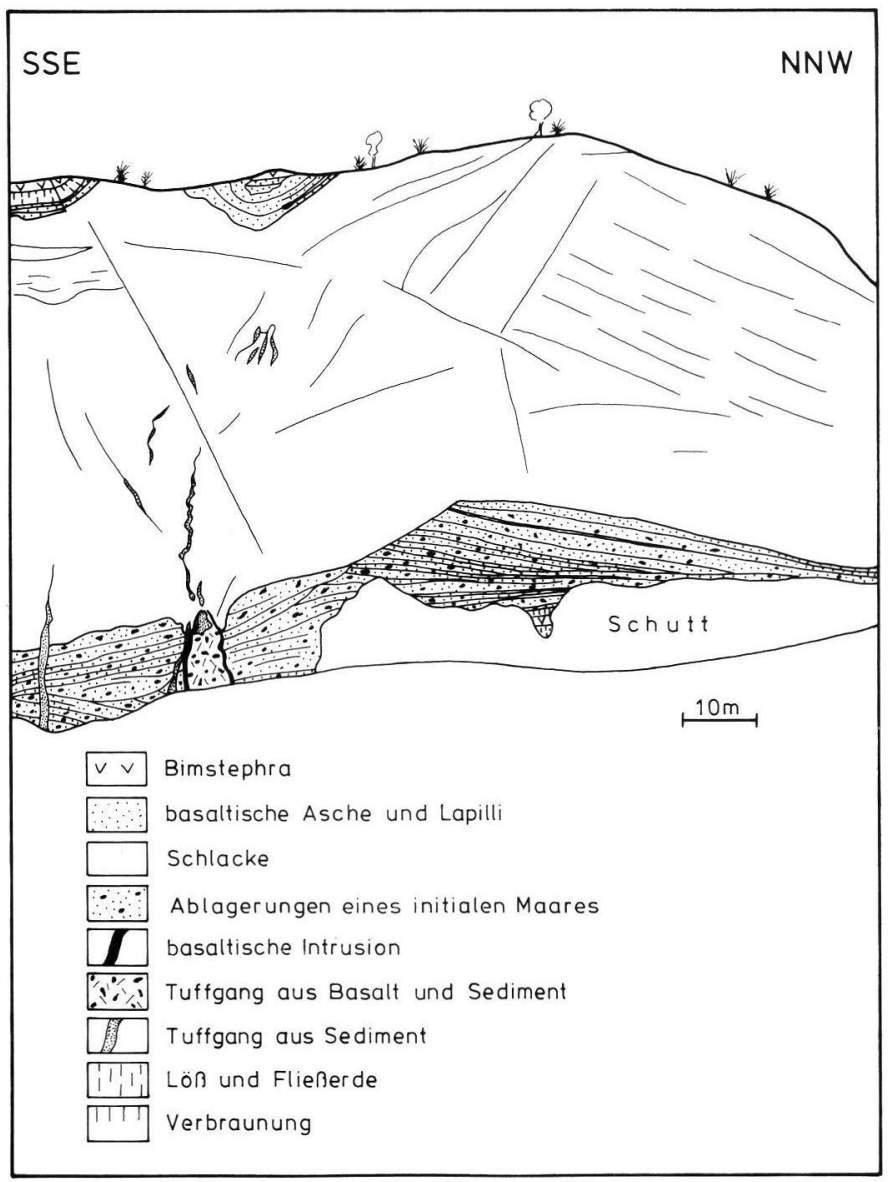

Abb. 3: Aufbau des Wannenköpfe-Schlackenkegels. Neben mindestens zwei Tuffringablagerungen und den Schlackenablagerungen existieren zahlreiche Tuffgänge und Basaltintrusionen. Die ehemaligen Kratermulden am Top des Schlackenkegels bildeten Sedimentfallen.

Fig. 3: The Wannenköpfe scoria cone consists of tuff deposits, the scoria cone, basaltic dykes and sediments injected into the main wall. The crater acted as sediment trap at beginning of penultimate glaciation.
Die Möglichkeit zur Erosion bestand erst, nachdem die Kratermulde mit Sediment gefüllt war. Das Profil $C$ liegt im Bereich der Maarablagerungen und der Basalt- und Tuffgänge. Das Profil D befindet sich teils in einer Mulde eines Nebenkraters und teils am südlichen Außenhang des kleinen Schlackenkegels.

\section{Eruptionsgeschichte der Wannenköpfe}

Einen kurzen Überblick über den Aufbau des Wannen-Schlackenkegelkomplexes geben BOGAARD \& SCHMINCKE (1990: 180f), SCHMINCKE, BOGAARD \& FREUNDT (1990: 105ff) und FRECHEN (1993: 114f).

Die Beschreibung des vulkanologischen Formenschatzes und die Rekonstruktion der Eruptionsgeschichte des etwa $60 \mathrm{~m}$ hohen Schlackenkegels bezieht sich auf den Bereich der Aufschlüsse A-D. Eine Detailbeschreibung weiterer Aufschlüsse der Wannenvulkangruppe sowie die petrographischen Untersuchungen hätten den Rahmen dieser Arbeit gesprengt.

\subsection{Phreatomagmatische Initialphase und Maarbildung}

Im Bereich der Wannenköpfe waren zur Zeit der Geländearbeiten (1992 und 1993) unterhalb des Schlackenkegelkomplexes der nordöstlichen Wannenköpfe (im Bereich der Profile $\mathrm{A}$ und $\mathrm{C}$ ) phreatomagmatische Ablagerungen mehrerer initialer Maare aufgeschlossen (Abb. 3). Die jüngsten Maar-Ablagerungen, als Profil „A. Maar" beschrieben, bestehen aus gut geschichteten, nebengesteinsreichen Tephren mit wechselnder, meist jedoch schlechter Sortierung.

Das Liegende der Wannenköpfe steht in einem kleinen Aufschluß unterhalb der Ablagerungen des initialen Maares in Profil A an. Dort sind kaltzeitliche Sedimente aufgeschlossen, die mit einer Verbraunung abschließen. Oberhalb einer Fließerde wurde eine bis zu $7 \mathrm{~cm}$ mächtige, parautochthone Bimstephra abgelagert. Der darüber folgende schwache Boden wird von einer $14 \mathrm{~cm}$ mächtigen, geschichteten Aschenschicht überlagert, an deren Basis vereinzelt Abdrücke von Gräsern und kleinen Blättern vorkommen. 


\section{Profil A. Maar}

Profilmächtigkeit: $16,69 \mathrm{~m}$

Hangendes: Schweißschlacken der Wannenköpfe

Liegendes: schwach humose Verbraunung (Abb. 6)

Höhe unter Beschreibung

Interpretation

GOK in $[\mathrm{m}]$

$-0,50 \quad$ Lapilli und Schlacke; schwarz

-2,50 Lapilli und Schlacke; rotbraun; vereinzelt Tonflatschen; schwach geschichtet

$-7,00 \quad$ Asche, Lapilli und Schlacke; grauschwarz;

Wechsellagerung; geschichtet

$-9,10 \quad$ Brockentuff; reich an Tonflatschen; geschichtet

-10,05 Lapilli; viele Nebengesteinsfragmente: Tonflatschen bis zu 1,40x0,60 m Größe

-10,20 Lapilli; an der Basis basaltische Bomben; sehr viele Nebengesteinsfragmente: bis zu 0,20 m große Tonflatschen, Schieferbröckchen sowie Quarz- und Quarzitgerölle

-11,50 Lapilli; bis zu 0,40 m große, basaltische Bomben; selten bis zu 0,10 m große Tonflatschen, Quarz- und Quarzitgerölle, Schieferbröckchen; graubraune, feinkörnige Matrix

$-13,70 \quad$ Brockentuff; bis zu 0,35 m große, basaltische Bomben; porenreiche Basaltbrekzien, viele gelbgrüne, schmutziggraue und rötliche, gefrittete Tonflatschen, viele Quarzund Quarzitgerölle; braune, braunrote, sedimentreiche Matrix -15,30 Lapilli; schwarz; im unteren Bereich etwas gröber; bis zu 0,30 $\mathrm{m}$ große, basaltische Bomben; wenige poröse Basaltbrekzien; rotbraune, gelbrote und graue bis zu $0,12 \mathrm{~m}$ große Tonflatschen, bis zu $4 \mathrm{~cm}$ große Quarz- und Quarzitgerölle sowie Schieferbröckchen; sedimentreiche, schmutzigbraune Matrix

$-16,00 \quad$ Wechsellagerung von sediment- und lapillireichen Lagen; bis zu $0,15 \mathrm{~m}$ große, wenig poröse Basaltklasten; schließt nach oben mit sandigen Lagen ab, die eine gradierte Schichtung aufweisen

$-16,15$ Schluff; sandig; graubraun: bis zu $1 \mathrm{~cm}$ große idiomorphe Augitkristalle, Schieferbröckchen, Quarz- und Quarzitgerölle, geschichtet

-16,31 Lapilli und Schlacke; relativ grob; schwarz; rötliche und graue, bis zu 3,5 m große Tonflatschen sowie Quarz- und Quarzitgerölle; bis zu 3,5 cm große Phlogopitkristalle

$-16,40 \quad$ Schluff; tonig, sandig, schwach kiesig; basaltische Lapilli, Quarz- und Quarzitgerölle, Schieferbröckchen, Augitkristalle; rötliche, graubraune Matrix

-16,46 Lapilli; bis zu 3,5 cm groß; wenige Quarz- und Quarzitgerölle, Schieferbröckchen

-16,54 Schluff; sandig tonig; lößlehmartig; glasreiche, schwarze bis zu $2 \mathrm{~cm}$ große Lapilli; ein 1,5 cm großes Bimslapilli; Schieferbröckchen

-16,58 Asche; feinkörnig; hellgrauschwarz

Fall-

Tuffit; vulkanoklastisches Sediment mit viel Aschenmaterial; verbacken

-16,69 Asche; graugrünlich; vereinzelt Abdrücke von Gräsern und kleinen Blättern an der Basis
Fallablagerung

Maar-

Ablagerungen ablagerung 
Die phreatomagmatischen Ablagerungen lassen eine gewisse Zunahme der Eruptionsdynamik und -energie während der Initialphase erkennen. Die vulkanische Aktivität beginnt zunächst mit der Eruption von lapilli-, aschen- und sedimentreichen Pyroklastika, die wenig poröse, bis zu $15 \mathrm{~cm}$ große, hydroklastische Basaltbrocken enthalten. Die basaltischen
Bomben haben keine ausgeprägten Einschlagstrichter gebildet, so daß ein ballistischer Transport mit großer Fallhöhe wenig wahrscheinlich ist.

Den Hauptanteil der Nebengesteinsfragmente bilden bunte, gefrittete Tonbrocken, Quarz- und Quarzitgerölle sowie devonische Schieferbrocken oder -bröckchen, aber auch dichtes, juveniles basaltisches

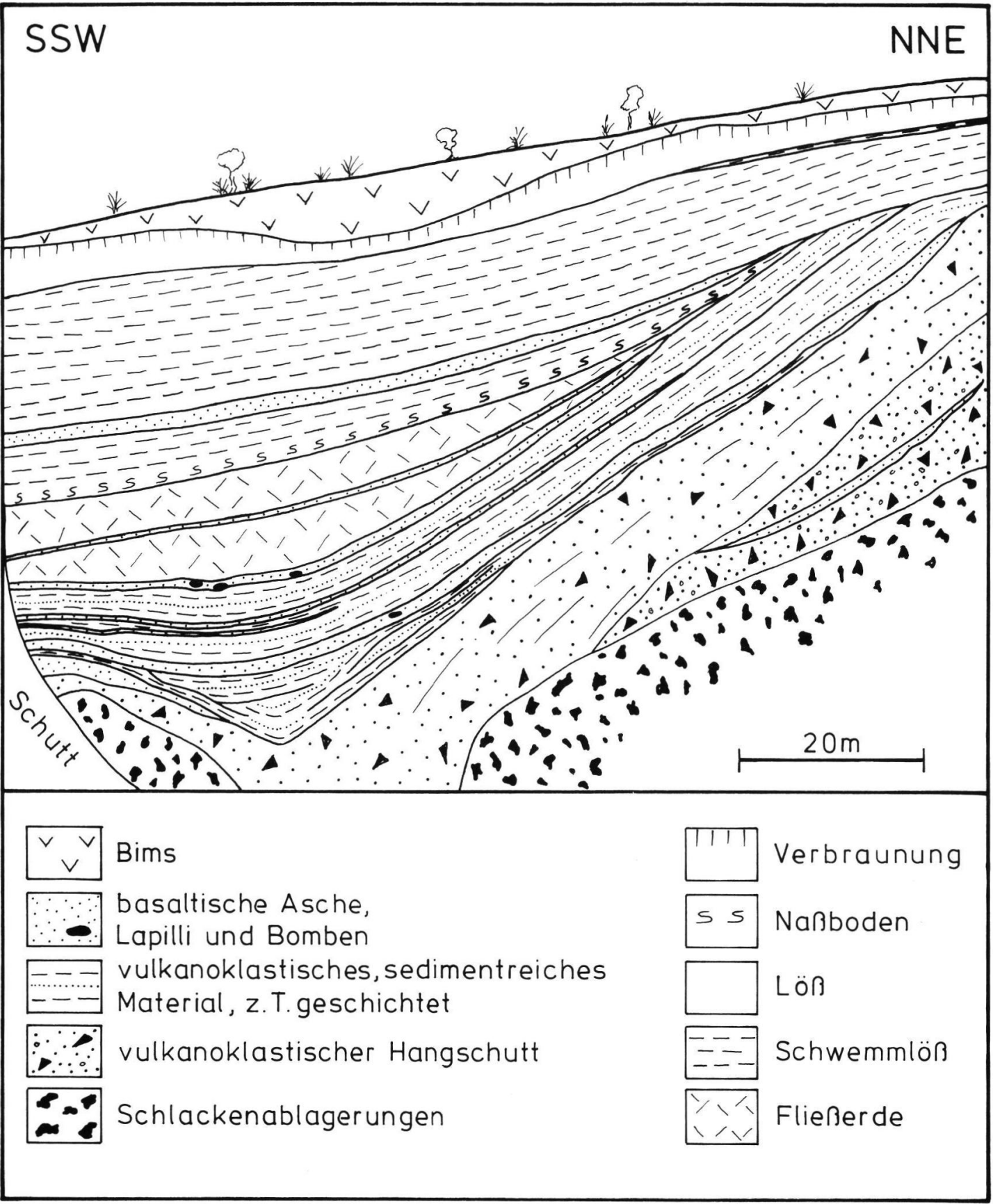


Material. Die Größe der Nebengesteinsfragmente alterniert zum Hangenden hin und gipfelt zweimal in brockentuffartige, geschichtete, aber schlecht sortierte Tephren. Die Größenzunahme der Nebengesteinsfragmente zum Hangenden hin weist auf zunehmende Heftigkeit der Eruption.

Die Wechsellagerungen von Asche und Lapilli sowie der zunehmende Anteil an juvenilem Schlackenmaterial im oberen Bereich der Maar-Ablagerungen deuten das Ende der Initialphase und den Übergang von phreatomagmatischem Eruptionsstil in eine strombolianische Schlackenwurftätigkeit an.

Innerhalb der jüngsten Maarbildungpshase, aber auch zwischen den phreatomagmatischen Tephren der beiden Maare konnten keine Hinweise auf längere zeitliche Unterbrechungen, z. B. in der Form von schwachen Bodenbildungen festgestellt werden.

Sowohl in der östlichen als auch in der westlichen Wannenvulkangruppe sind mehrfach phreatomagmatische Ablagerungen initialer Maare unterhalb der Schlackenkegel im Sinne von Lorenz (1986: 267) vorhanden. So waren zur Zeit der Geländeaufnahme in den Jahren 1992 und 1993 Reste von mindestens 6 Maaren in der östlichen Wannenvulkangruppe aufgeschlossen. Vergleichbare Ablagerungen finden sich in den Schlackenkegelkomplexen Eiterköpfe, Schweinskopf-Karmelenberg sowie Tönchesberg und, wie bereits SCHMINCKE (1977: 17 und 19) angibt, für den Kunkskopf und Rothenberg.

Für die Entstehung von Tuffringen und Maare und den damit verbundenen phreatomagmatischen Eruptionen nimmt SCHMINCKE (1977: 33) eine Wechselwirkung zwischen Magma, Oberflächen- und Grundwasser an. Dabei kommt es aufgrund von Wasserdampf zu explosiven Eruptionen, bei denen nebengesteinsreiche, schlecht sortierte, aber relativ gut geschichtete Tephren abgelagert werden.

\subsection{Strombolianische Schlackenwurftätigkeit}

In der zweiten Phase wurden hauptsächlich heiße, noch plastisch verformbare Lavafetzen strombolianisch gefördert. Diese mäßig bis schwach verfestigten Schlacken, z. T. Schweißschlacken, erreichen Längen von 2-3 m sowie Dicken von 0,15-0,30 m und sind zum Teil lagig angeordnet. Sie deuten auf hohe Temperaturen und damit auf eine relative Nähe der Ablagerung der Schlacken zum Krater hin. Der Fremdgesteinsgehalt der Schlacken ist gering. Untergeordnet kommen gefrittete Tone, devonische Schiefer und vereinzelt Quarz- und Quarzitgerölle vor. In den Wannenköpfen sind an der NordwestWand des nördlichen Steinbruchs phreatomagmatische Ablagerungen den Schweißschlacken zwischengeschaltet.

Die vulkanischen Schlacken stellen neben den effu- siv geförderten Lavaströmen das Hauptförderprodukt des Schlackenkegelkomplexes dar. SCHMINCKE, BOgAaRd \& Freundt (1990: 107) wiesen an den Schlacken eine chemische Differenzierung des Magmas nach, einen Übergang von einer frühen tephritischen in eine späte basanitische Zusammensetzung.

Durch das zunehmende Höhenwachstum des Schlackenkegels und das Übersteilen der Hänge wurden die Flanken des Schlackenkegels instabil, und es kam in der Endphase der Eruption sowie postvulkanisch zu Hangrutschungen. Im Profil D (Abb. 4) sind mehrere Lagen mit vulkanoklastischem Detritus aufgeschlossen. Der Hangschutt ist mäßig bis schlecht sortiert, unverfestigt und kann in mehrere Einheiten untergliedert werden. Die Schichteinheiten sind im Profil D zum Teil linsig ausgebildet und keilen rasch aus.

\subsection{Finalphase mit Intrusionen von Tuff- und Basaltgängen}

In der Spätphase intrudierten zahlreiche Basalt- und Tuffgänge sowohl in die Maar- als auch in die Schlackenablagerungen (BOGAARD \& SCHMINCKE 1990: 180). Ähnliche Beobachtungen existieren für eine Reihe weiterer Schlackenkegel der Osteifel. So wurden unter anderem am Herchenberg (NolL 1967), am Schweinskopf-Karmelenberg und am Tönchesberg derartige Intrusionen beschrieben (FRECHEN, unveröffentlicht).

An den Wannenköpfen existieren mehrere klastische Tuffgänge, die zahlreiche, in der Schlackenbrekzie blind endende Apophysen bilden. Die klastischen Tuffgänge bestehen aus rötlichem, gelblichem und gräulichem, tonigem Material, das stellenweise schwach sandig und verfestigt ist. Der Durchmesser der klastischen Tuffgänge variiert zwischen 0,50 $\mathrm{m}$ und wenigen Zentimetern, wobei sich die Gänge nach oben verjüngen. Mindestens ein derartiger Tuffgang erreichte die damalige Oberfläche im Gipfelbereich des Schlackenkegels (Profil B, Abb. 3). Die finale Phase wird von SCHMincke, BOGAard \& FREUnDT (1990: 109) als Schlammfontänentätigkeit mit der Bildung eines kleinen Schlammsees gedeutet. Die Entstehung von Schlammfontänen im Topbereich des Schlackenkegels ist aufgrund des akuten Wassermangels und des sehr porösen Untergrundes, das Schlackenmaterial stellt kein Wasserstauer dar, schwierig vorzustellen und überinterpretiert. Statt dessen ist eine starke hydrothermale Zersetzung von Material des Kraterbodens und tuffitischen Materials, das durch die Förderung eines hochfluidisierten, mobilen Gemischs aus Wasserdampf und vulkanoklastischem, sedimentreichem Material während der Finalphase wesentlich wahrscheinlicher. 
In der nur wenige Zehnermeter entfernten Kratermulde (Profil D) sind keine ziegelroten Tone nachweisbar, jedoch abgespültes feinschluffig-toniges, vulkanoklastisches Material.

Die Ablagerungen dieser Finalphase sind in einer mehr als $1 \mathrm{~m}$ mächtigen, verfestigten, z. T. ziegelroten Tonschicht an der Basis der kleinen Kratermulde aufgeschlossen. Die Beschreibung des Tons erfolgt weiter unten (siehe Profil B). Schlammfontänen mit größeren Auswurfhöhen sind dagegen eher unwahrscheinlich, da derartige Ablagerungen an der Basis von Profil D fehlen.

Neben den klastischen Tuffgängen existieren basaltische Gangintrusionen, die bis in das obere Drittel des Schlackenkegels reichen. Der grauschwarze Basalt ist sehr dicht und hat eine zum Teil glasartige Matrix. Aus Profil C wurde bereits von SCHMINCKE, BOGAARD \& FREUNDT (1990: 107) ein komplexer Tuffgang beschrieben, so daß hier nur eine kurze $\mathrm{Zu}-$ sammenfassung der Beobachtungen erfolgt. Die komplexe Intrusion (Abb. 3) wird an beiden Seiten von einem etwa $0,60 \mathrm{~m}$ breiten, sehr dichten, porenarmen, glasartigen Basalt umgrenzt. Dazwischen sind basaltische, brekziöse bis zu $20 \mathrm{~cm}$ große Basaltklasten, die glasartig und sehr dicht sind, in einer tonigen, schwach sandigen, rötlichen, verfestigten Matrix enthalten. An der Basis (August 1993) ist der komplexe Tuffgang etwa $6 \mathrm{~m}$ breit.

Die Genese von Tuffgängen wird allgemein bei NoLL (1967: 105) und speziell für die komplexe Intrusion der Wannenköpfe bei SCHMINCKE, BOgAaRD \& FreundT (1990: 107) beschrieben. Durch die Intrusion von Magma in die liegenden, grundwasserreichen Tone und Sande wurde das Grundwasser aufgeheizt, und es entstand ein hochfluidisiertes und -mobiles Gemisch aus Wasserdampf, Sediment und abgeschreckten, glasartigen, basaltischen Brocken. Das aufsteigende Magma blieb an der Grenze Maar/Schlackenablagerungen stecken. Die Basaltfragmente wurden in einer Suspension aus Wasserdampf und tonig/feinschluffigem Material in die Maarablagerungen injiziert. Der Tuffgang reicht mit dem klastischen Material bis in die Schlackenablagerungen, endet dort aber blind.

\subsection{Effusive Phase}

Im Bereich der Profile A-F ist $70 \mathrm{~m}$ nördlich von Profil E ein Lavastrom in einer schwer zugänglichen Geländedepression aufgeschlossen. Der Lavastrom hat eine Breite von etwa $40 \mathrm{~m}$ bei einer Mächtigkeit von maximal $5 \mathrm{~m}$ und besteht aus gut gesäultem, dichtem Basalt. Eine Schicht aus schwarzen Lapilli (Äquivalent der Tephren B-1 und D-1) liegt unterhalb des Lavastroms. Inwieweit der Lavastrom zu den an der Ostseite der Wannenköpfe vorhandenen, ausgedehnten Lavaströmen beitrug, ist aufgrund der Aufschlußverhältnisse nicht mehr feststellbar.

\subsection{Tephra-Ablagerungen benachbarter Eruptionszentren}

Oberhalb der Schlackenablagerungen und des vulkanoklastischen Hangschutts folgen plinianisch und phreatomagmatisch abgelagerte Aschen und Lapilli. Das den Fallablagerungen - basanitische und tephritische Aschen und Lapilli - zwischengeschaltete pyroklastische Material hat eine sehr dunkle, feinkörnige Matrix. Die dunkle Farbe stammt von einem hohen Anteil an basaltischen Lapilli- und Aschenresten. Die Beschreibung der einzelnen Tephren erfolgt weiter unten (Profil B und D).

Zwei Aschenlagen werden von BOGAARD \& SCHMincKe (1990: 178) aufgrund ihrer chemisch-mineralogischen Zusammensetzung den Tephren des Plaidter Hummerichs (Tephra D-6) und einem unbekannten tephritischen Eruptionszentrum (Tephra D-7) zugeordnet. Durch die tephrochronologischen und lößstratigraphischen Untersuchungen sind Korrelationen mit Tephralagen aus weiteren Osteifel-Kraterprofilen, speziell denen des Tönchesberges, möglich.

\section{Sedimentologisch-pedologische Ergebnisse/Profilbeschreibungen}

Die sedimentologisch-pedologischen Ergebnisse beruhen auf Profilbeschreibungen der Jahre 1992 und 1993. Die Profile A, B, C und D bleiben mittelfristig zugänglich. Für ausgewählte Proben aus Profil A wurden Schlämmanalysen nach KöHN durchgeführt. Der organische Kohlenstoffgehalt und der Karbonatgehalt wurden für Sedimentproben aus den Profilen A, B und D coulometrisch mit einem Coulomat 702 der Firma Ströhlein analysiert. Aus der Differenz von tc- (gesamter Kohlenstoffgehalt) und toc-Gehalt (organischer Kohlenstoffgehalt) wurde der Karbonatgehalt kalkuliert. Bei den Messungen wurde eine Abhängigkeit zwischen tc- und toc-Gehalt festgestellt, so daß die toc-Werte korrigiert werden mußten. Der daraus resultierende Corg-Wert ist ein Kriterium für die relative Anreicherung von organischem Kohlenstoff im Sediment. Die Werte des organischen Kohlenstoffs (Corg) wurden im Sinne von BoENIGK, FreCHEN \& WeIDENFELLER (1994) berechnet. Die relative Abnahme der toc-Gehalte und der Corg-Werte aufgrund von Alteration seit der Ablagerungszeit wurden bei dieser Korrektur nicht berücksichtigt. Die höchsten toc-Werte ergaben sich aus Analysen von humosen saalezeitlichen Sedimenten.

Bodenmikromorphologische bzw. mikromorphologische Untersuchungen an ausgewählten Horizonten ergänzten die Profilbeschreibungen und die coulometrischen Analysen. 


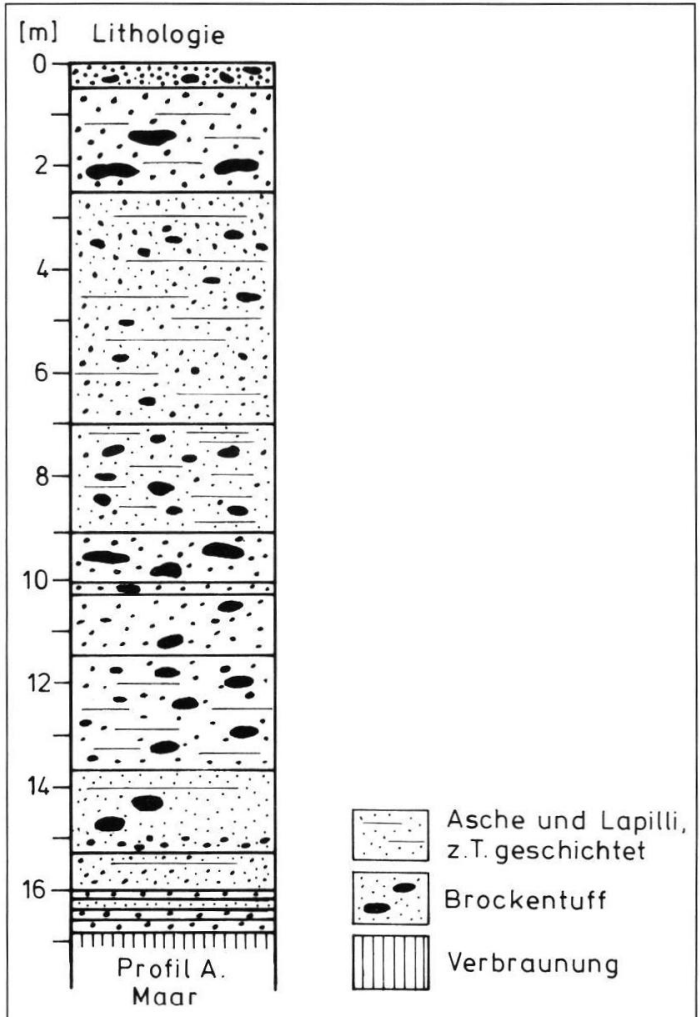

Abb. 5: Säulenprofil der Maar-Ablagerungen (Profil A. Maar) oberhalb von Profil A.

Fig. 5: Idealised section of the phreatomagmatic deposits of the initial phase of the Wannenköpfe volcanism (Profil A. Tuffring).

\section{Profil A}

Hangendes: Tephra der Initialphase (siehe Profil A. Maar)

Profilhöhe: 2,29 m, davon 1,20 m erbohrt

Höhe unter Beschreibung

Interpretation

GOK in $[\mathrm{m}]$

$-0,25$ Schluff; schwach tonig und sandig; dunkelbraun, schokoladenbraun; obere $10 \mathrm{~cm}$ bröckelig; vereinzelt Bimsbröckchen;

Verbraunung schwach humos; Verbraunung; im Kontaktbereich zum darüber liegenden Tuff Abdrücke von Gräsern und kleinen Blättern

-0,33 Bimstephra; bis $3.5 \mathrm{~cm}$ große Lapilli; Schieferbröckchen; Matrix aus schwach sandigem und tonigem Schluff; mäßig sortiert; Bims greift taschenförmig in die darunter liegende Fließerde; parautochthon

Diskordanz $-0,43$

Schluff; schwach tonig; gelbbraun; Verbraunung nimmt nach unten ab; Bims- und Schieferbröckchen $(<0,5 \mathrm{~m} \varnothing)$,

Diskordanz $-0,83$

Diskordanz $-1,08$

Schluff; tonig, schwach kiesig; vereinzelt bis zu $4 \mathrm{~cm}$ große Quarz- und Quarzitgerölle; gelbbraun, nach oben hin heller Fließerde werdend; wenige Mn-Flecken; keine vulkanischen Komponenten

Schluff; gelbgraubraun; vereinzelt bis zu $5 \mathrm{~cm}$ große Lößkindl; Kalkmycel; karbonatisch

$-2,29$

\subsection{Profil A}

Das Liegende des Wannenköpfe-Schlackenkegelkomplexes ist derzeit unterhalb des Maares in Profil A aufgeschlossen (Abb. 3 und 5). Die Profilbeschreibung erfolgt jeweils vom Liegenden zum Hangenden.

Die Abfolge beginnt mit einem äolisch abgelagerten Löß, der einen Tongehalt von 13\% und ein Maximum der Kornverteilung im Mittel- und Grobferbröckchen in die zunehmend toniger werdende Fließerde eingearbeitet. Es handelt sich um einen Tonanreicherungshorizont, dessen Tongehalt 23,1\% beträgt. Dieser B-Horizont ist nahezu vollständig entkalkt. In den Dünnschliffen zeigt sich ein Vesikelgefüge mit ungleichmäßig verteilten, bis zu 80 ım dicken Tonbelägen sowie Mn- und Fe-Flecken. Die Grobkomponenten bestehen aus Quarzen und sehr selten Pyroxenen. Direkt unterhalb der Bimsen zum Hangenden hin zu. In den Dünnschliffen eigt sich ein Vesikelgefüge mit bis zu $5 \mathrm{~mm}$ großen Hohlraumfüllung vorhanden. In der Grundmasse zu $40 \mu \mathrm{m}$ dicke Mn- und Fe-Flecken vorhanden. 


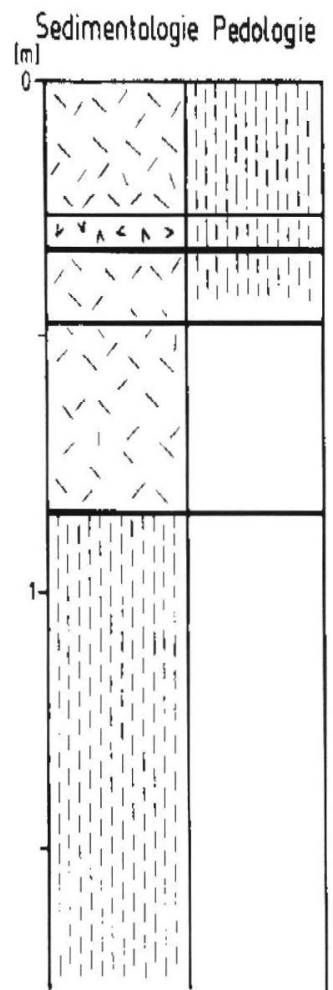

Profil A
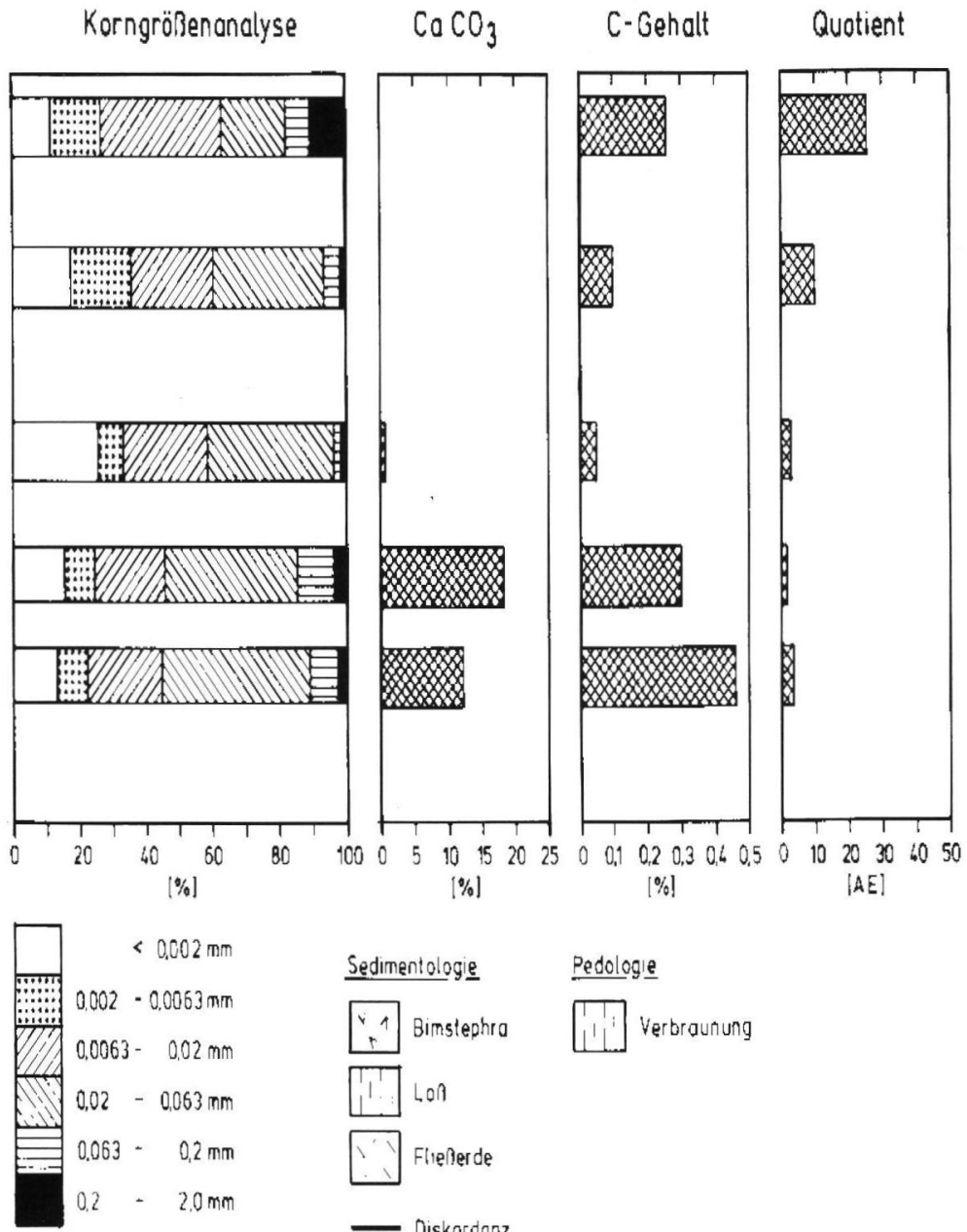

Sedimentologie
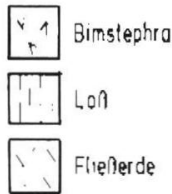

Diskardanz
Pedologie

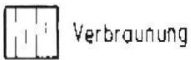

Abb. 6: Sedimentologisch-pedologische Ergebnisse von Profil A. Korngrößenbestimmungen, Karbonatgehalt, organischer Kohlenstoffgehalt (toc) und relative Humusanreicherung (Corg).

Fig. 6: Sedimentological and pedological results of section A. Grain size distribution, carbonate content, organic carbon content (toc) and relative humic enrichment (Corg).

Die 2-7 cm mächtige Bimsschicht greift zum Teil taschenförmig in die darunter liegende Fließerde ein. Die Tephra ist parautochthon abgelagert und leicht vermischt mit schluffigem, lößartigem Sediment. Der Anteil der Bimslapilli nimmt nach oben hin rasch ab. Das Profil schließt mit einer interstadialen Bodenbildung ab. Der Tongehalt ist mit 11,8 \% nicht erhöht, während der Grobsandanteil wegen der eingearbeiteten Bimslapilli recht hoch ist. Die Humusanreicherung dieser Verbraunung drückt sich in dem toc-Gehalt von $0,26 \%$ und einem Corg-Wert von 26 aus. Im Dünnschliff zeigt sich ein Vesikelgefüge. Tonbeläge sind kaum sichtbar. Bis zu $0,5 \mathrm{~mm}$ große vulkanische Komponenten sind häufig in der Grundmasse vorhanden, des weiteren Olivin- und Pyroxenkristalle. Die vulkanischen Reste sind zum Teil eisenumkrustet. Die Hohlräume sind röhrenförmig angeordnet. Die Porosität des verbraunten Sedimentes ist geringer als bei den bereits diskutierten Schliffen. An der Unterkante der Tephra finden sich vereinzelt Abdrücke von Gräsern und/oder kleinen Blättern. Darüber folgt die Initialphase mit den bereits beschriebenen Maar-Ablagerungen.

\subsection{Profil B}

Das Profil B befindet sich mit einer Mächtigkeit von insgesamt 9,39 $\mathrm{m}$ in einer kleinen Kratermulde im Gipfelbereich der nordwestlichen Wannenköpfe. 


\section{Profil B}

Profilmächtigkeit: 9,39 m

Liegendes: Schweißschlacken der Wannenköpfe

Höhe unter Beschreibung
GOK in $[\mathrm{m}]$

$-0,30 \mathrm{~m} \quad$ Bimstephra; Lapilli; im oberen Bereich umgelagert

$-0,45 \quad$ Schluff; schwach tonig; graubraun, schokoladenbraun; prismatisches Gefüge;

$-0,60$

$-1,10$

$-1,80$

$-2,72$

$-3,06$

$-4,73$

$-4,95$

$-5,19$

$-5,44$

$-5,54$

$-5,68$

$-5,73$

$-5,81$

$-6,70$

$-6,76$

$-6,86$

$-7,38$

Lapilli; grauschwarz; ungeschichtet; sehr wenige kleine
Schluff; hellgraubraun; Verbraunung nimmt nach unten ab

Schluff; gelbgraubraun; sehr viel vulkanoklastisches

Material; karbonatisch

Schluff; gelbgraubraun; karbonatisch

Lapilli; häufig Quarz- und Quarzitgerölle; grünlich, grauschwarz; umgelagert

Lapilli; bis zu 0,80 m große basaltische Bomben mit Ein-

schlagstrichter; schwarz; durch rötlich, braunes, sedimentreiches

Band zweigeteilt

Lapilli; schluffig, schwach tonig; dunkelgelbbraun; geschichtet;

karbonatisch; viele Nebengesteinsfragmente (Ton- und Schiefer-

bröckchen, Quarz- und Quarzitgerölle, bis zu 1,5 cm große

Phlogopitkristalle)

Lapilli; sandig, schluffig; schwarz, graubraun; zweigeteilt durch

schluffigen, graubraunen Sand

Schluff; schwach sandig; wenig Aschenmaterial; sekundäre

Karbonatausfällungen in Gängen

Asche; grauschwarz; sedimentreich; geschichtet

Schluff; sandig, schwach tonig und kiesig; dunkelgelbbraun; hoher

Anteil an vulkanischem Material, bis zu $2,5 \mathrm{~cm}$ große Schieferbröckchen,

Lapilli, meist kleiner als $1 \mathrm{~cm}$; grauschwarz; selten rote Tonbröck-

chen und kleine Quarz- und Quarzitgerölle

Schluff; tonig, sandig; dunkelgelbbraun, reich an feinkörnigem, vulkanoklastischem Material

Lapilli und Asche; grauschwarz; feinkörnig $(<3 \mathrm{~mm} \varnothing)$; zum Teil

lößummantelt; umgelagert

Schluff; schwach tonig; dunkelolivgrün, graubraun, dunkelgraubraun; nach oben hin heller werdend und zunehmend sekundäre Aufkalkung in bis zu $1 \mathrm{~cm}$ breiten Röhrchen; Rhizolenien; porös; oberster Bereich schwach humos

Linsen von basaltischer Lapilli, bis zu $80 \mathrm{~cm}$ breit und bis zu $6 \mathrm{~cm}$ mächtig; umgelagert

Schluff; schwach tonig und sandig; dunkelolivgrün; zementiert;

sekundär aufgekalkt; weiße diffuse Karbonatflecken

Brockentuff; etwas gröbere grauschwarze Lapilli; im unteren

Bereich Bomben aus bis zu 0,60 m großen Tonflatschen mit

Einschlagstrichter; hoher Nebengesteinsanteil: bis zu $4 \mathrm{~cm}$ große Quarzund Quarzitgerölle; selten Schieferbröckchen; Grenze zur darunter

liegenden Tephra bildet ein an Karbonat angereicherter und

karbonatisch zementierter Horizont; geschichtet

Nebengesteinsfragmente

Ton; schluffig; dunkelgrau; bröckelig zerbrechend; sekundär aufgekalkt

Asche; schwarzgrau; vereinzelt Augitkristalle; karbonatisch zementiert

Ton; schluffig; dunkelgraubraun; verbacken; sekundär aufgekalkt

Ton; ziegelrot; sehr spröde und hart; keine Schichtung; weiße

karbonatische und schwarze Mineralausfällungen (Manganoxide)

auf Kluftflächen besonders im unteren Bereich.
Tephra B-8

[LST]

Pararendzina

[Alleröd-Boden]

Bv-Bereich

Fließerde

Löß

Fließerde

Tephra B-7

Tephra B-6

Tephra B-5

Tephra B-4

Tephra B-3

Tephra B-2

Tephra B-1

Ton

Tephra B-0 
Die Deckschichtenfolge des Profils B beginnt mit einem verfestigten, ziegelroten Ton sowie weiteren dunkelgraubraunen Tonen. Im ziegelroten Ton ist kein Karbonat vorhanden. Der darüber liegende dunkelgraubraune Ton ist sekundär aufgekalkt und hat einen $\mathrm{CaCO}_{3}$-Gehalt von 16,0\% (s. Abb. 7).
4,9\%, in denen zum Teil Linsen basaltischer Lapilli enthalten sind, folgt ein dunkelgraubrauner, dunkelolivgrüner Schluff. Nach oben hin sind zunehmend mit Karbonat ausgekleidete ehemalige Wurzelgänge mit Durchmesser bis zu $1 \mathrm{~cm}$ vorhanden. Der $\mathrm{CaCO}_{3}$-Gehalt liegt hier bei $13,8 \%$. In diesem

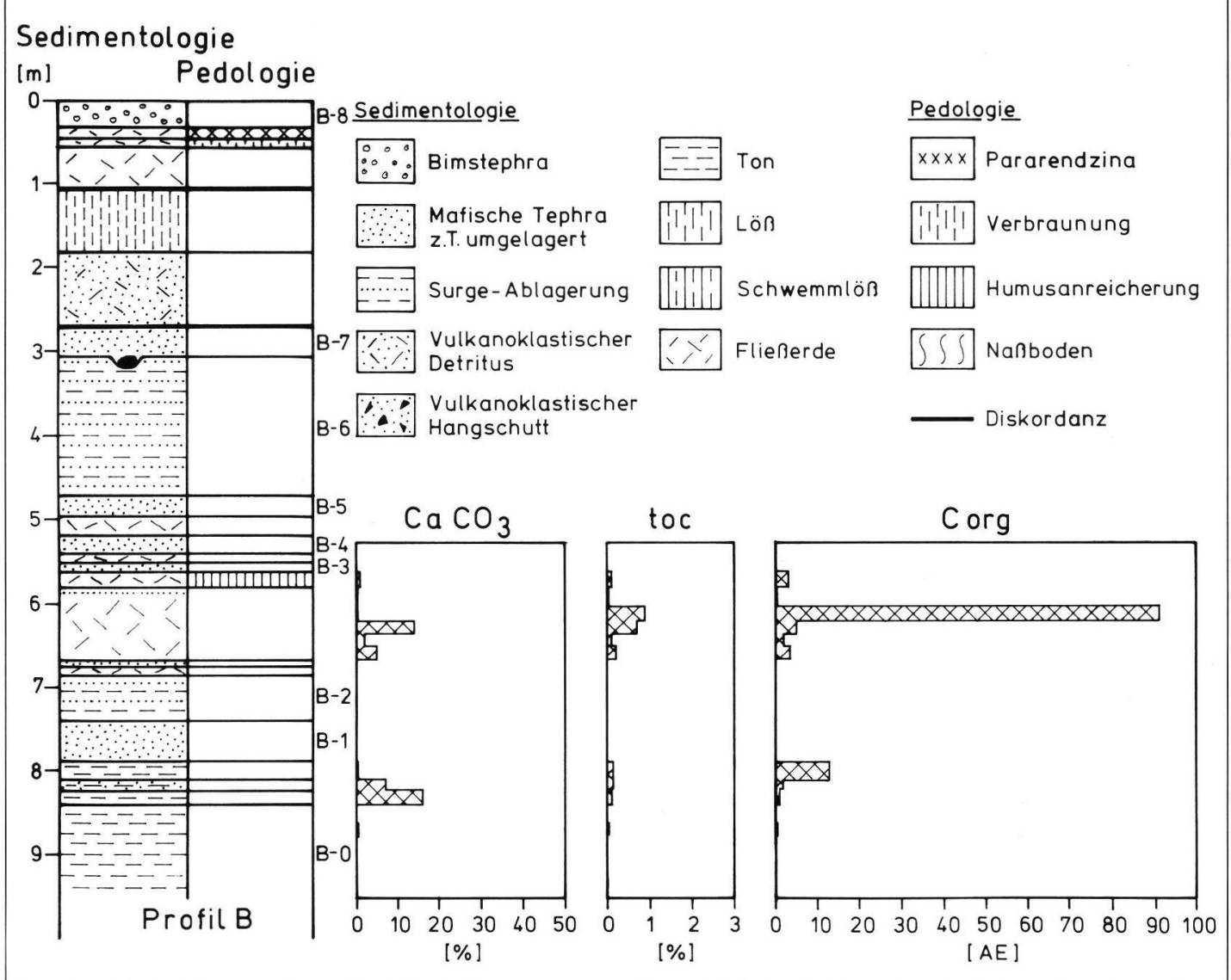

Abb. 7: Sedimentologisch-pedologische Ergebnisse von Profil B. Karbonatgehalt, organischer Kohlenstoffgehalt (toc) und relative Humusanreicherung (Corg).

Fig. 7: Sedimentological and pedological results of section B. Karbonate content, organic carbon content (toc) and relative humic enrichment (Corg).

Bei den in der Sedimentfalle abgelagerten Tephren handelt es sich um typische Fallablagerungen (Tephren B-1, B-3, B-4, B-5, B-7 und B-8), die mit Ausnahme der Tephra B-8, dem Laacher See-Bims, aus mafischen Aschen oder Lapilli bestehen. Die Tephren sind überwiegend in situ abgelagert. Es kommen ebenfalls umgelagerte Aschen vor, z. B. oberhalb der Tephra B-2. Basaltische Bomben finden sich an der Basis der Tephra B-7. Phreatomagmatischer Eruptionsstil ist durch die Tephren B-2 und B-6 dokumentiert. Auch dort sind vulkanische Bomben, hauptsächlich aus Tonflatschen vorhanden. Oberhalb von umgelagerten schluffigen, schwach tonigen Sedimenten mit einem $\mathrm{CaCO}_{3}-\mathrm{Gehalt}$ von
Sedimentpaket kommt vulkanischer Detritus makroskopisch nur untergeordnet vor. Unterhalb der Tephra B-3 sind die lehmigen, dunkelgelbbraunen Sedimente fast vollständig entkalkt. Die lehmigen, dunkelgelbbraunen Sedimente sind jedoch schwach humos mit einem maximalen Corg-Wert von 91 (toc $=0,91 \%$ ), so daß für diese Schicht eine Humusanreicherung und damit eine schwache Bodenbildung angenommen werden muß.

Oberhalb der Tephra B-7 folgt eine Fließerde, die sehr viel vulkanoklastisches Material enthält, und schließlich äolischer Löß. Das Profil schließt mit dem $\mathrm{Bv}$ - und Ah-Horizont des Alleröd-Bodens und der Tephra B-8 ab. 


\subsection{Profil D („Canyonprofil“)}

Im „Canyonprofil“ sind etwa $20 \mathrm{~m}$ Decksedimente in einer Depression oberhalb des vulkanoklastischen Hangschutts abgelagert worden. Die Profilbeschrei- bung erfolgte an den leicht verfolgbaren Tephrahorizonten jeweils um 5 - 10 m horizontal nach Süden versetzt.

Das Säulenprofil ist aus 5 Teilprofilen kombiniert (Abb. 8).

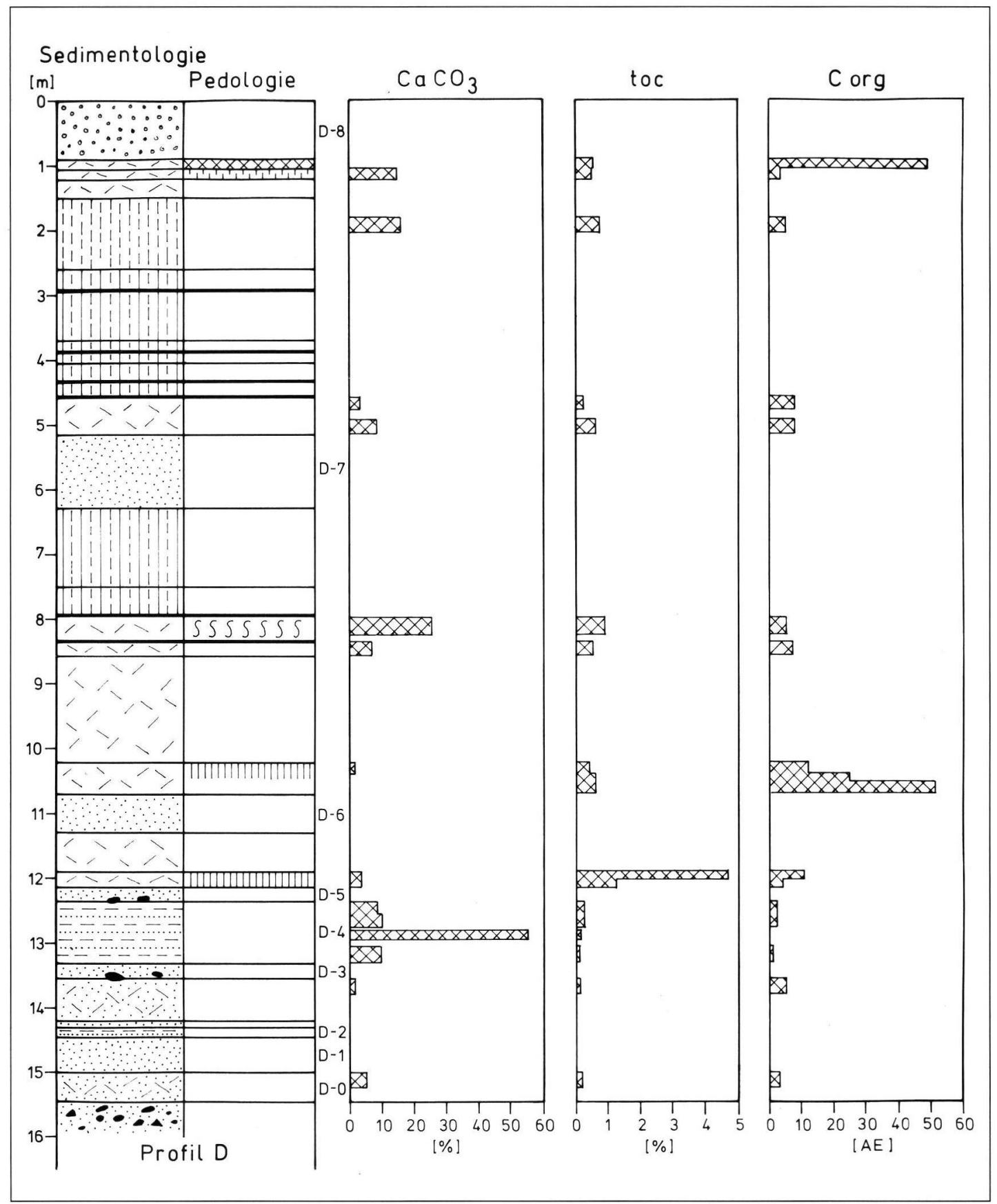

Abb. 8: Sedimentologisch-pedologische Ergebnisse von Profil D. Karbonatgehalt, organischer Kohlenstoffgehalt (toc) und relative Humusanreicherung (Corg).

Fig. 8: Sedimentological and pedological results of section D. Carbonate content, organic carbon content (toc) and relative humic enrichment (Corg). 


\section{Profil D}

Profilhöhe: $15,27 \mathrm{~m}$

Liegendes: vulkanoklastischer Hangschutt der Wannenköpfe

$\begin{array}{lll}\text { Höhe unter } & \text { Beschreibung } & \text { Interpretation } \\ \text { GOK in }[\mathrm{ml} & \end{array}$

\begin{tabular}{ll}
\hline$-0,90$ & $\begin{array}{l}\text { Bimslapilli; umgelagerte, abgeschobene Reste des Laacher } \\
\text { See-Bimses }\end{array}$ \\
$-1,05$ & $\begin{array}{l}\text { Schluff; schwach tonig; braun, schokoladenbraun; pris- } \\
\text { matisch brechend }\end{array}$ \\
$-1,20$ & $\begin{array}{l}\text { Schluff; schwach tonig; braun; Verbraunung } \\
-1,50\end{array}$ \\
& Schluff; schwach sandig; hoher Anteil an vulkanischem \\
$-2,60$ & Material, bis zu 4 cm große Lapilli; häufig Augitkristalle \\
$-2,95$ & Schluff; hellgelbbraun; Kalkmycel; stark karbonatisch \\
& Schluff; sandig; gelbbraun; häufig sandige Einschaltungen; \\
viele Augitkristalle; schwach geschichtet
\end{tabular}

\author{
Tephra D-8 \\ Pararendzina \\ [Alleröd-Boden] \\ Bv-Bereich \\ Fließerde \\ Löß \\ Schwemmlöß
}

\section{Diskordanz \\ $-3,69$ \\ $-3,89$ \\ Diskordanz \\ $-4,04$ \\ $-4,34$ \\ Schluff; selten Linsen aus sandigem Material; hellgelbbraun; \\ Kalkmycel; karbonatisch; schwach geschichtet \\ Schluff; sandig; Sandkomponente enthält sehr viele kleine Augitkristalle; \\ gelbbraun; vereinzelt kleine Quarz- und Quarzitgerölle; geschichtet; \\ Schluff; gelbbraun; vereinzelt feinkörniger, vulkanoklastischer \\ Detritus; karbonatisch \\ Schluff; schwach sandig; nach oben hin weniger Aschenmaterial; \\ gelbgraubraun, nach oben etwas heller werdend; karbonatisch; \\ schwach geschichtet; Kiesschnüre mit überwiegend vulkano- \\ klastischem Material; Augitkristalle}

Diskordanz

$-4,54$

Schluff; nach oben hin weniger vulkanoklastischer Detritus;

gelbgraubraun; karbonatisch; Kiesschnüre aus feinkörnigem vulkano-

klastischem Material

Diskordanz

$-5,14$

Schluff; schwach sandig; gelbgraubraun; hoher Aschen-

anteil; seltener Lapilli; Kiesschnüre; karbonatisch

$-6,28$

Asche und Lapilli; grauschwarz; umgelagert; geschichtet;

Wechsellagerung von Aschen- und Lapilli mit gering-

mächtigen sedimentreichen Lagen; die unteren $10 \mathrm{~cm}$ ent-

halten Lapilli, die oberen $8 \mathrm{~cm}$ Asche

$-7,50 \quad$ Schluff; sandige, braune Bänder; gelbgraubraun;

karbonatisch; z. T. geschichtet

$-7,95$ Schluff; schwach sandig; Wechsellagerung von schluffig/

schluffig-sandigen Lagen; aus umgelagerter vulkanischer Asche

und Löß; selten Lapilli; gelbgraubraun

Diskordanz

$-8,38$

Schluff; sehr wenige kleine Steinchen; fahlgrau; nach oben

hin etwas gelbbrauner; karbonatisch

$-8,58$

$-10,18$

Schluff; sandig, sandige Lagen; gelbgraubraun; karbonatisch

Schluff; sandig, schwach kiesig; Solifluktionsschutt; graubraun;

Schwemmlöß

Lößkomponente nimmt nach oben hin zu; die unteren $33 \mathrm{~cm}$ enthalten sehr viel Asche und Lapilli

-10,23 Schluff; sandig, kiesig; gelbbraun; viel Asche und Lapilli; schwach

karbonatisch

-10,71 Schluff; sandig, kiesig; häufig Asche und Lapilli; manchmal

linsenförmig angereichert; dunkelgraubraun; die oberen $26 \mathrm{~cm}$

sind dunkler und humoser; nach oben feinkörniger werdend;

schwach karbonatisch; Schichtung löst sich von unten nach

Fließerde

Tephra D-7

[Tephritische

Tephra]

Schwemmlöß

oben auf

Humusanrei-

Naßboden

Fließerde

cherung

Fließerde 
-11,28 Asche; grauschwarz; umgelagert; geschichtet; mindestens 6 geringmächtige Lagen mit lößartigem Sediment zwischengeschaltet

-11,92 Sand; schluffig; nach oben hin gröber werdend; viele Quarzund Quarzitgerölle und Schlackenreste; schwach karbonatisch; geschichtet

-12,14 Schluff; sandig; hoher Anteil an Asche und Lapilli; grauschwarzbraun (humusfarben) nach oben hin heller werdend; Kalkmycel; karbonatisch; 3-4 cm große Holzkohleflitter; zementiert -12,38 Lapilli; grauschwarz; stellenweise umgelagert; zieht z. T. beulenartig in das darunterliegende Sediment hinein; häufig vulkanische Bomben; Lapillilage durch $1-2 \mathrm{~cm}$ mächtiges Sedimentband zweigeteilt

-13,33 Schluff; sandig, schwach kiesig; graubraun, gelbgraubraun, braun; karbonatisch; Wechsellagerung von schluffig-sandigen Lagen; im unteren Drittel $2 \mathrm{~cm}$ mächtige Bank aus Karbonat; im oberen Drittel geschichtet; besteht überwiegend aus Nebengestein: gefrittete Tonbröckchen, die z. T. mit Karbonat umkrustet sind; oberer Bereich ist schluffig und schwach sandig, leicht gebändert, wenige Schlackenreste Asche; die unteren $5 \mathrm{~cm}$ in situ; darüber umgelagerte und schwach geschichtete Asche; sedimentreiche, karbonatische Zwischenlage

-14,29 Schluff; tonig, sandig; hoher Anteil an Asche und Lapilli; graubraun; schwach karbonatisch in pseudomycelartigen weißen Gängen; im untersten Bereich häufig Lapilli; lößummantelte Lapilli

-14,32 Lapilli; schwarz, umgelagerte Tephra D-1

-14,47 Sand; kiesig; hoher Anteil an Quarz- und Quarzitgeröllen; Lapilli sind oft lößummantelt; umgelagert;

-14,99 Lapilli; schwarz; in situ; keine Schichtung; bläuliche Eisenmineralüberzüge; sehr geringer Nebengesteinsanteil

$-15,27$ Schluff; sandig; schwach tonig; bis zu $15 \mathrm{~cm}$ große Schlackenreste; dunkelgraubraune, nicht karbonatische, zementierte Matrix; hoher Anteil an Asche und Lapilli Tephra D-3

Tephra D-6

Fließerde

Humusanreicherung

Tephra D-5

Tephra D-4

\author{
(1)
}

vulkanoklastischer Hangschutt

Tephra D-2

Tephra D-1

Tephra D-0

Schlacke
Die Decksedimente beginnen oberhalb von vulkanoklastischem Hangschutt wie im Profil B zunächst mit einer pyroklastischen Abfolge. Aufgeschlossen sind zum Teil umgelagerte Fallablagerungen aus gut sortierten mafischen Aschen und Lapilli (D-1, D-3, D-5, D-6, D-7 und D-8) sowie phreatomagmatische Ablagerungen (D-2 und D-4). Die Fallablagerungen der Tephra D-5 sind durch basaltische Bomben mit Einschlagstrichtern an der Basis charakterisiert. Die Nebengesteinskomponenten der phreatomagmatischen Ablagerungen im "Canyonprofil" sind deutlich kleiner als die der Maarablagerungen an der Basis der Wannenköpfe. Es handelt sich dabei um distale Fließablagerungen phreatomagmatischer Eruptionen.

Die Abfolge beginnt mit feinkörnigen, vulkanoklastischen Sedimenten, in denen sich bis zu $15 \mathrm{~cm}$ große Schlackenreste finden, die synsedimentär vom Kraterrand in die Depression gerutscht sind. Das Material ist in der Muldenmitte deutlich mächti- ger als hangaufwärts (Abb. 4). Die vulkanoklastischen Sedimente sind schlecht sortiert und zeigen keine Schichtung. Die Sedimente sind sekundär aufgekalkt und haben einen $\mathrm{CaCO}_{3}$-Gehalt von $4,3 \%$. Es handelt sich dabei um distale Ablagerungen der Finalphase, dessen Zentrum in der Kratermulde von Profil B zu finden ist.

Im Dünnschliff ist ein Schwammgefüge mit einer Porosität von 20-30\% sichtbar. In der Grundmasse finden sich Quarze, große Pyroxene und Olivine sowie Schlackenstückchen. Die Matrix der Grundmasse besteht aus Ton. Die vulkanischen Minerale sind zum Teil mit Tonbelägen umkrustet. Die Schlackenstückchen weisen oft bis zu $20 \mu \mathrm{m}$ dicke Verwitterungsbeläge aus Eisenoxiden auf, stellenweise existieren Fe-Nester. Die Eisenmobilisation, die einen Teil der Verbraunung und Verbackung bewirkt hat, wird auf Eisenumsatz aus den mafischen Mineralen der Aschen und Lapilli zurückgeführt. Sowohl die Ton- als auch die Eisenumkrustung gehen nicht auf 
Bodenbildungsprozesse zurück. Statt dessen handelt es sich dabei um in die Kratermulde umgelagerten, feinkörnigen, vulkanoklastischen Detritus der finalen Phase. Die Dünnschliffauswertung sowie die toc- und Corg-Werte sprechen eindeutig gegen eine Humusanreicherung sowie Bodenbildung in diesem Bereich.

Darüber folgt ein geringmächtiges Band umgelagerter, schwarzer Lapilli, die oft lößummantelt sind, und ein weiterer $0,35 \mathrm{~m}$ mächtiger, sandiger, schwach toniger und schwach karbonatischer Schluff mit einem hohen Anteil an Lapilli im unteren Bereich. Der Corg-Wert liegt bei 5 . Im Dünnschliff zeigt sich ein Schwammgefüge. Olivin, Pyroxen, Sparit und bis zu $300 \mu \mathrm{m}$ große Schlackenstückchen finden sich als Grobkomponenten. In der schwach tonigen Grundmasse sind viele bis zu $100 \mu \mathrm{m}$ große Fe- und MnNester vorhanden. Es finden sich Fe-Beläge um Schlackestückchen, selten auch um Olivinkristalle. Die Porosität liegt bei etwa 20\% und beruht hauptsächlich auf röhrenförmigen Hohlräumen.

Im unteren Drittel der Tephra D-4 ist eine $2 \mathrm{~cm}$ mächtige Karbonatbank zwischengeschaltet, deren $\mathrm{CaCO}_{3}$-Gehalt $54,7 \%$ beträgt. Der Corg-Wert ist dagegen sehr gering und liegt nur bei 0,3 hat. Oberhalb und unterhalb der Karbonatbank der Tephra D-4 liegt der $\mathrm{CaCO}_{3}$-Gehalt zwischen 8 und 9\%.

Zum Hangenden hin folgt ein sandiger, grauschwarzer Schluff mit viel vulkanischem Material in Asche- und Lapilligröße. Nach oben hin wird die Farbe etwas heller, und es finden sich pseudomycelartige Strukturen und $3-4 \mathrm{~cm}$ große Holzkohlestückchen. Die Fließerde ist schwach karbonatisch und hat einen Corg-Wert von 10,8.

Oberhalb dieser Humusanreicherung ist eine nach oben hin gröber werdende Fließerde abgelagert worden. Die Fließerde hat einen hohen Anteil an kleinen Schlackenresten sowie Quarz- und Quarzitgeröllen. Darauf folgt eine teilweise umgelagerte Asche (Tephra D-6), die wiederum von einer Fließerde, die hauptsächlich aus umgelagertem, nach oben hin feinkörniger werdendem vulkanoklastischem Material besteht. Die Schichtung löst sich von unten nach oben zunehmend auf. Die obersten $26 \mathrm{~cm}$ der fast vollständig entkalkten Fließerde sind dunkler und humoser. Die Corg-Werte, die zwischen 24,9 und 54 variieren, deuten auf eine weitere Humusanreicherung und somit auf eine zweite schwache Bodenbildung. Im Bodendünnschliff ist ein humoses Schwammgefüge feststellbar. An Grobkomponenten finden sich hauptsächlich Schlackenstückchen. Darüber folgen fließerdeartige Sedimente mit Solifluktionsschutt, z. T. mit sandigen Lagen. Der Lößanteil nimmt nach oben hin zu.

Darauf folgt ein fahlgrauer, karbonatischer Schluff, der als Naßboden angesprochen wird. Der Naßboden hat einen Karbonatgehalt von 15,2\% und einen
Corg-Wert von 5,2. Oberhalb des Naßbodens wurde wieder Schwemmlöß abgelagert, dessen Karbonatgehalt deutlich niedriger ist.

Oberhalb der tephritischen Tephra D-7 folgt eine schwach karbonatische Fließerde mit Kiesschnüren. Nach oben hin nimmt die Anzahl der Diskordanzen zu. Zahlreiche Kiesschnüre und abgeschwemmtes, lößartiges Material mit feinkörnigem, vulkanoklastischem Detritus dominieren. Der Schwemmlöß hat einen Karbonatgehalt von 13,1 bis 14,6\%

Eine Pararendzina, der Alleröd-Boden, schließt die Löß-/Paläobodenabfolge nach oben hin ab. Der Corg-Wert ist deutlich erhöht und liegt bei 49 (toc $=0,49 \%$ ). Die Abfolge endet mit zum Teil abgeschobenem Laacher See-Bims (Tephra D-8).

\section{Diskussion}

Die vulkanische Abfolge und der Eruptionsverlauf der Wannenköpfe sind typisch für den Aufbau von Osteifel-Schlackenkegelkomplexen. Dies wurde bereits von SCHMINCKE, BOGAARD \& FREUNDT (1990: 105) und BOGAARD \& SCHMINCKE (1990: 180f) erwähnt.

Die östliche Wannengruppe ist nach SCHMINCKE, BoGAARD \& FREUNDT (1990: 108) in einen älteren westlichen und einen jüngeren östlichen Schlackenkegelkomplex untergliedert. Dies kann aufgrund der Deckschichtenfolge und den zwischengeschalteten Tephren bestätigt werden.

An den Wannenköpfen deutet die pyroklastische Abfolge auf einen schnellen Eruptionsverlauf ohne bedeutende zeitliche Unterbrechungen. Für die Dauer des Ausbruchs sind Tage, Wochen oder Monate anzunehmen. Zwischen den beiden Maar-Bildungsphasen ist ebenfalls keine längere zeitliche Lücke nachweisbar. Es sind weder Humusanreicherungen noch Verbraunungen vorhanden.

Im Profil A (Abb. 6) unterhalb der Maar-Ablagerungen sind zunächst eindeutig kaltzeitliche Sedimente abgelagert, u. a. äolisch abgelagerter Löß und eine Fließerde, in der es zu einer schwachen Tonanreicherung gekommen ist. Darauf folgt eine parautochthon abgelagerte Bimstephra, die von BOGAARD et al. (1989: 525) mit der Hüttenberg-Tephra korreliert wird. Die darüber liegende Verbraunung mit schwacher Humusanreicherung reicht nicht aus, um die Tonanreicherung in der Fließerde zu erklären. Für die Hüttenberg-Tephra wurde ein $40 \mathrm{Ar} / 39 \mathrm{Ar}-$ Durchschnittsalter von $215 \pm 4$ ka kalkuliert (BoGAARD et al. 1989: 525). Dieses Alter impliziert einen Ausbruch der Bimstephra nach dem ersten vorletztinterglazialen Klimaoptimum, das würde den Sauerstoffisotopenstadien 7b (230-219 ka) oder 7a (219$190 \mathrm{ka})$ im Sinne von Martinson et al. (1987: 20f) entsprechen. Die Löß-/Paläoboden-Abfolge aus Profil A unterstützt diese Annahme. Es ist wichtig zu bemerken, daß es sich bei dem Tonanreicherungs- 


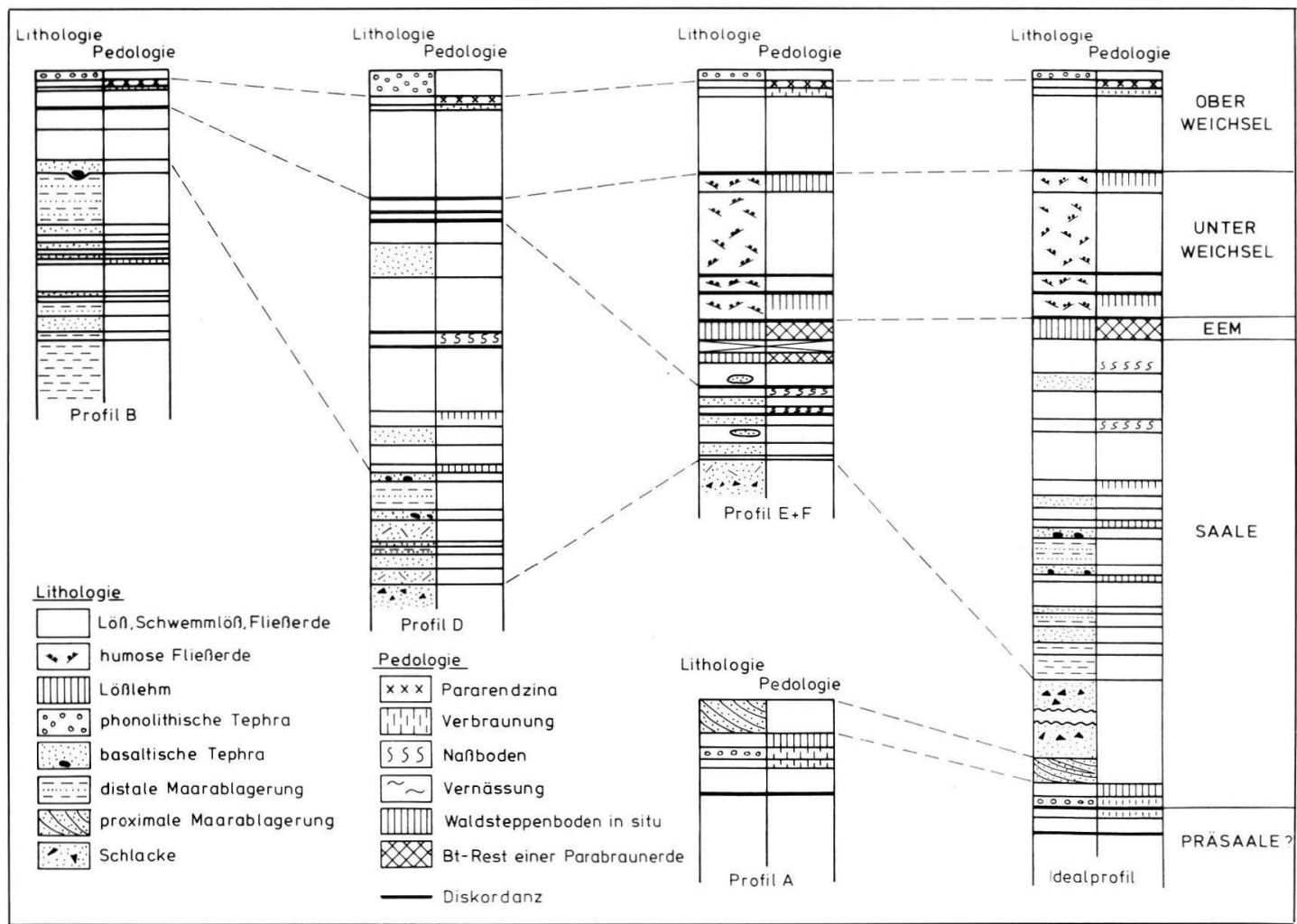

Abb. 9: Korrelation und Kombination der Profile A, B., D und E sowie daraus resultierendes idealisiertes Profil.

Fig. 9: Correlation of section A, B, D and $\mathrm{E}$ and resulting idealised section of Wannenköpfe loess paleosol sequence.

horizont nicht um den Bt-Rest einer Parabraunerde handelt, ein Al-Horizont fehlt ebenfalls. Daraus folgt, daß die Hüttenberg-Tephra entweder am Ende der drittletzten Kaltzeit, wie von BOGAARD et al. (1989: 523) postuliert, oder zu Beginn des vorletzten Glazials eruptiert wurde (s. Abb. 9). Die schwach humose Verbraunung oberhalb der Bimstephra deutet auf interstadiale Verhältnisse am Beginn des vorletzten Glazials. Dies würde zeitlich dem Übergang der Sauerstoffisotopenstadien von 7 a nach 6 entsprechen (MARTINSON et al. 1987: 20f). Ob die warmzeitlichen Sedimente des vorletzten Interglazials erodiert sind, kann aufgrund der Diskordanz nicht entschieden werden. Der Ah- und der Al-Horizont, die für die Tonanreicherung der Fließerde verantwortlich sind, fehlen ebenso.

Weitere schwache Humusanreicherung sind in zumindest drei Fällen den Tephren der Deckschichtenfolge zwischengestaltet. Oberhalb der Tephra D-6 wurden kleine Blattabdrücke (mündl. Mitt. A. Justus) gefunden. Die gleichbleibende Mächtigkeit der pedogen überprägten Schichten sowie die schwache Humusanreicherung deuten auf einen relativ kurzen Zeitraum für die Bodenüberprägung. Derartige interstadiale Böden sind während der letzten 200.000 Jahre nur aus dem Beginn der letzten und vorletzten Kaltzeit bekannt. Da den Deckschichten oberhalb der Tephra D-7 eine Parabraunerde sowie Waldsteppenböden zwischengeschaltet sind, liegt es nahe, diese Bodenbildung mindestens mit dem Beginn des vorletzten Glazials zu korrelieren. Die vorläufigen physikalischen Datierungen widersprechen dem nicht. In Analogie der vorletztglazialen Abfolge bei Bibus (1974) werden diese schwach humosen, vulkanischen Böden als frühsaalezeitliche Interstadiale oder als ein frühsaalezeitliches Interstadial interpretiert, das durch die vulkanischen Ablagerungen in mehrere schwache Bodenbildungsphasen aufgespalten ist.

Eine ähnliche Interpretation geben BOGAARD \& SCHMINCKe (1990: 181), jedoch werden die Ablagerungen oberhalb der Hüttenberg-Tephra als Böden eines Interglazials interpretiert. Dies kann durch die Geländeaufnahme und die sedimentologisch-pedologischen Ergebnisse nicht bestätigt werden. Für eine Ablagerung der Hüttenberg-Tephra am Ende der drittletzten Kaltzeit bzw. zu Beginn der vorletzten Warmzeit vor etwa 245 ka gibt es keine Belege. Die ${ }^{40} \mathrm{Ar} / 39 \mathrm{Ar}$-Daten und die humusreichen Verbraunungen unterhalb des Maares sowie in den Kratermulden deuten auf eine Entstehung der Wannenköpfe unter interstadialen Bedingungen zu Beginn der vorletzten Kaltzeit. 
WindHeuser (1977) versuchte das Alter der Wannenköpfe über eine Korrelation der Maar- mit den Brockentuff-Ablagerungen des Mittelpleistozänprofils Kärlich weiter einzugrenzen. Eine Korrelation der beiden Tephren ist aufgrund der phreatomagmatischen Eruptionsdynamik jedoch ausgeschlossen. Bei phreatomagmatischen Eruptionen kommt es durch den horizontalen Transport sehr rasch zu einer Mächtigkeits- und Korngrößenabnahme. Ein rasches Ausdünnen der Schichten ist an den Wannenköpfen bereits auf $80 \mathrm{~m}$ zu beobachten (Abb. 3), so daß ein Transport von brockentuffartigem Material aus Kärlich über eine Entfernung von $7,5 \mathrm{~km}$ ausgeschlossen werden kann. Ähnliche Ablagerungen finden sich unterhalb der Schlackenkegelkomplexe Schweinskopf-Karmelenberg, Tönchesberg, Kunkskopf und Rothenberg. Wie bereits erwähnt, handelt es sich um die Initialphasen-Ablagerungen eines Schlackenkegels.

Die ${ }^{40} \mathrm{Ar} /{ }^{39} \mathrm{Ar}$-Datierungen an Dunkelglimmern aus Schlacken der Wannen mit $235 \pm 35 \mathrm{ka}$, der Eiterköpfe mit $213 \pm 40 \mathrm{ka}$ und des Tönchesberges mit $202 \pm 14$ ka sind vorläufige Ergebnisse (BOGAARD \& SCHMINCKE 1990: 178).

Oberhalb der Schlackenbrekzie liegen in der Kratermulde von Profil D weitere basanitische und tephritische Tephren, darunter die basanitische Tephra des
Plaidter Hummerichs und die tephritische Fallablagerung eines unbekannten Eruptionszentrums. Die ${ }^{40} \mathrm{Ar} / 39 \mathrm{Ar}-\mathrm{Datierung}$ der Korretsberg-Asche mit $243 \pm 65 \mathrm{ka}$ ist ebenfalls vorläufig, die Plaidter Hummerich-Tephra hat ein $40 \mathrm{~K} / 39 \mathrm{Ar}$-Alter von $238 \pm 20 \mathrm{ka}$ (SCHMINCKe \& MERTES 1979: 614). Aus den vorläufigen

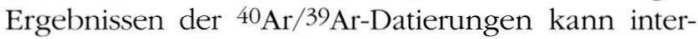
pretiert werden, daß die Wannenvulkangruppe, der Plaidter Hummerich, der Korretsberg und der Tönchesberg unter interstadialen klimatischen Verhältnissen vor etwa 200 ka oder weniger als 200 ka eruptiert sind, d. h. zu Beginn des vorletzten Glazials.

Im Profil B findet sich der erste Hinweis auf schwache Humusanreicherung unterhalb der Tephra B-3, im Profil D oberhalb der Tephra D-5. Das bedeutet, daß die klimatischen Bedingungen mäßig kalt waren, so daß es zu einer schwachen Bodenbildung und damit zu Humusanreicherungen kommen konnte.

Am Tönchesberg sind derartige Klimaanzeiger und Bodenbildungen unterhalb des Eembodens nicht vorhanden (BOENIGK \& FRECHEN im Druck). Eine tephrochronologische Korrelation ermöglicht jedoch eine Kombination der beiden Profile, so daß unsere paläoklimatischen und paläoökologischen Interpretationen um die frühglazialen, saalezeitlichen $\mathrm{Hu}-$ muszonen erweitert werden (Abb. 10).

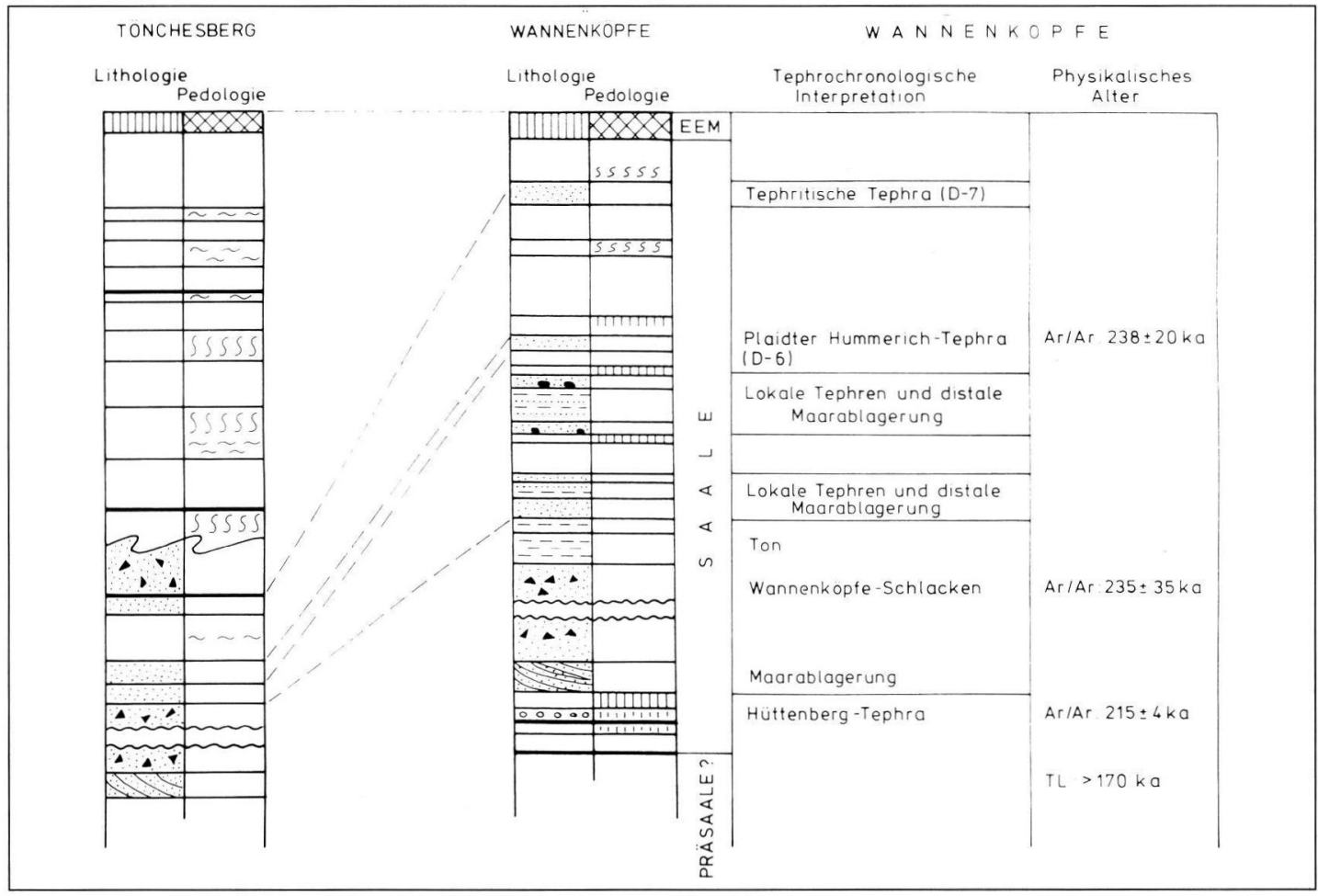

Abb. 10: Korrelation des idealisierten Löß-/Paläoboden-Profil der Wannenköpfe mit dem des Tönchesberges aus BoENIGK \& FRECHEN (im Druck) für die vorletztglazialen Abfolgen.

Fig. 10: Correlation of penultimate loess paleosol sequences of Wannenköpfe and Tönchesberg. 
Die Löß-/Paläobodenabfolge der Wannenköpfe ist oberhalb der tephritischen Tephra stark reduziert. Es treten zahlreiche Kiesschnüre verknüpft mit Diskordanzen auf. Saalezeitliche Naßböden, wie sie am Tönchesberg und Schweinskopf-Karmelenberg aufgeschlossen sind, fehlen hier ganz.

Eine Parabraunerde oder unterweichselzeitliche Humuszonen sind weder im Profil B noch D vorhanden. In einem weiter südlich gelegenen Steinbruch werden die Deckschichten in den Profilen $\mathrm{E}$ und $\mathrm{F}$ (Abb. 2) von einem rotbraunen Bt-Rest einer Parabraunerde untergliedert, die mit dem Eem-Boden korreliert wird (FRECHEN 1993: 114f). Unterhalb der Parabraunerde sind 3 Tephren den periglazialen Sedimenten (Profile E und F) zwischengeschaltet. Diese Tephren können mit denen der Profile B und D korreliert werden (Abb. 9). Oberhalb des Eem-Bodens lagern typische unterweichselzeitliche Ablagerungen, darunter zwei Waldsteppenböden sowie humose Fließerden und Lehmbröckelsande. Die darüber folgenden Lösse sind ungegliedert. Obwohl die Deckschichten stark reduziert sind, finden sich deutliche Parallelen zu der unterweichselzeitlichen Abfolge des Tönchesberges.

Eine Korrelation mit dem Profil Tönchesberg ist somit aufgrund lößstratigraphischer und tephrochronologischer Untersuchungen möglich. Vergleicht man die Ablagerungen, so folgt daraus, daß im Profil $\mathrm{C}$ des Tönchesberges, das sich am nördlichen Außenhang des Schlackenkegelkomplexes befindet, die saalezeitlichen, frühglazialen Ablagerungen fehlen. Unterhalb der Profile A und B des Tönchesberges sind die Decksedimente zwischen der tephritischen Asche und der Asche des Plaidter Hummerichs in mehreren Metern Mächtigkeit aufgeschlossen, derzeit aber nicht zugänglich. Im Profil C folgt am Tönchesberg über den Tephren des Korretsberges und des Plaidter Hummerichs lößartiges Material (Fließerden und Schwemmlösse), oberhalb der tephritischen Tephra eine vulkanoklastische Fließerde sowie die typische jüngere saalezeitliche Abfolge mit Lössen, Schwemmlössen und zwischengeschalteten Naßböden.

Die saalezeitliche Abfolge von Profil C des Tönchesberges wird durch die vorletztglazialen Sedimente in den Aufschlüssen der Wannenköpfe ergänzt und erweitert. Unterhalb der tephritischen Tephra konnten an den Wannenköpfen zwei schwach humose Horizonte nachgewiesen werden. Während der vorletzten Kaltzeit sind derartige Horizonte nur aus dem Beginn des Saale-Glazials bekannt.

Eine Kombination der Profilbereiche mittels tephrochonologischer Korrelation führt zu einer Ergänzung und Vervollständigung der saalezeitlichen Abfolge und damit zu einer genaueren zeitlichen Einstufung der untersuchten Osteifel-Schlackenkegelkomplexe. Für die untersuchten Schlackenkegel folgt, daß zunächst der Wannenköpfe-Schlackenkegelkomplex ausgebrochen ist. Die an den Wannenköpfen den Tephren zwischengeschalteten schwachen Humusanreicherungen deuten auf kurze Eruptionspausen von weniger als 1000 Jahren. Da die Tephra des Plaidter Hummerichs und des Korretsberges direkt auf dem Kraterboden des Tönchesberges aufliegen, folgt daraus, daß beide Vulkane kurze Zeit nach dem Ausbruch des Tönchesberges eruptierten.

Für die Schlackenkegelkomplexe Wannenköpfe, Eiterköpfe, Tönchesberg, Korretsberg und Plaidter Hummerich folgt insgesamt, daß sie zu Beginn der vorletzten Kaltzeit unter interstadialen Verhältnissen ausgebrochen sind.

\section{Dank}

Die Deutsche Forschungsgemeinschaft unterstützte die Untersuchungen im Rahmen des DFG-Projektes "Quartär der Eifel" (Bo413/5-2 und -3). Herrn Jörg Geßner danke ich für den Hinweis auf die "Schlammvulkanablagerungen“. Frau Dr. Antje Justus wies mich auf die gut gegliederte Löß-/Paläobodenabfolge hin. Den Herren Prof. Dr. W. Boenigk, Dr. H. Noll und Dr. Becker-Haumann sowie den Mitarbeitern der Abteilung Quartärgeologie danke ich für wertvolle Hinweise bei der Diskussion der Ergebnisse. Herrn Helsper, von der Rheinischen Provinzial Basal- und Lavawerke $\mathrm{GmbH}$, danke ich für die Erlaubnis zu Arbeiten im Bereich der Wannenköpfe. Die Reinzeichnungen wurden dankenswerter Weise von Frau Krings durchgeführt.

\section{Schriftenverzeichnis}

AHORNER, L. (1983): Historical seismicity and present-day microearthquake activity of the Rhenish Massif, Central Europe. - In: K. Fuchs (Hrsg.): Plateau Uplift; 198-211; Springer-Verlag Berlin.

AHrens, W. (1932): Die Basaltvulkane des südöstlichen Laacher-See-Gebietes und ihre Lavaströme. - Jahrbuch Preuß. Geol. L.-amt, 53: 851-878; Berlin.

Boecker, M. Lehmann, E. von \& Remy, H. (1972): Über eine Wirbeltierfauna aus den jüngsten würmzeitlichen Ablagerungen am Michelberg bei Ochtendung/Neuwieder Becken. - Decheniana, 124: 119-134; Bonn.

Boenigk, W. \& Frechen, M. (im Druck): Mittel- und oberpleistozäne Deckschichten des Tönchesberges, Osteifel. - In: SchIRMER, W. (Hrsg.): Landschaftsgeschichte im europäischen Rheinland. - Rheinlandverlag, Köln.

BOGAard, P. v. d. \& SCHMINCKe, H.-U. (1990): Die Entwicklungsgeschichte des Mittelrheinraumes und die Eruptionsgeschichte des Osteifel-Vulkanfeldes. - In: SCHIRMER, W. (Hrsg.): Rheingeschichte zwischen Mosel und Maas. - deuqua-Führer 1: 166-190; Hannover.

- Hall, C. M., Schmincke, H.-U. \& YORK, D. (1989): Precise singlegrain ${ }^{40} \mathrm{Ar} / 39 \mathrm{Ar}$ dating of a cold to warm climate transition in Central Europe. - Nature, 342: 523525; London. 
DECHEN, H. von (1964): Geognostischer Führer zu dem Laacher See und seiner vulkanischen Umgebung. Verlag Max Cohen \& Sohn, 596 S., Bonn.

FRECHEN, M. (1993): Eruptionsgeschichte und Deckschichtenfolge des Schlackenkegelkomplexes der Wannenköpfe in der Osteifel. - Nachrichten der Deutschen Geologischen Gesellschaft, 50: 114-115; Hannover.

- (1994): Thermolumineszenz-Datierungen an Lössen des Tönchesberges aus der Osteifel. - Eiszeitalter und Gegenwart, 44: 79-93; Hannover.

- \& Weidenfeller, M. (1994): Die mittel- und oberpleistozänen Deckschichtenfolge im Naturschutzgebiet „Eiszeitliches Lößprofil“ in Koblenz-Metternich. - Mainzer Geowissenschaftliche Arbeiten,

Justus, A., UrmersbaCH, K.-H. \& URMERSBACH, A. (1987): Mittelpaläolithische Funde vom Vulkan „Wannen“ bei Ochtendung, Kreis Mayen-Koblenz. - Archäologisches Korrespondenzblatt, 17: 409-417;

Kolfschoten, T. van \& ROTH, G. (iom Druck): Mittel- und spätpleistozäne Mollusken und Kleinsäuger aus Vulkanfüllungen des Osteifel-Gebietes, Deutschland. Jahrbuch Römisch-Germanisches Zentralmuseum,

LORENZ, V. (1986): On the formation of maars and diatremes. - Bulletin Vocanology, 48: 265-274; Berlin.

Noll, H. (1967): Trachytische Tuffgänge am Herchenberg bei Burgbrohl (Laacher-See-Gebiet) und der Erupti- onsmechanismus der Laacher Bimsvulkane. - Geologisches Institut der Universität zu Köln, Sonderveröffentlichungen, 13: 95-108; Köln.

- (1975): Die Geologie des Bausenberg-Vulkanes (Laacher Vulkangebiet). - Beiträge Landespflege Rheinland-Pfalz, Beiheft, 4: 15-32; Oppenheim.

Quiring, H. (1936): Erläuterungen zur Geologischen Karte von Preussen 1:25000, Blatt 5610 Bassenheim; Berlin.

SCHMINCKE, H.-U. (1977): Phreatomagmatische Phasen in quartären Vulkanen der Osteifel. - Geologisches Jahrbuch, Reihe A 39: 3-45; Hannover.

- BogaARd, P. v. d. \& Freundt, A. (1990): Quaternary Eifel Volcanism.- IAVCEI, International Volcanological Congress Mainz 1990; Excursion 1AI; 1-190; Witten.

SEMmel, A. (1991): Die Lößdecke des Plaidter Hummerich (Neuwieder Becken) und Fragen ihrer regional-stratigraphischen Einordnung. - Geologisches Institut der Universität zu Köln, Sonderveröffentlichungen, 82: 283-292; Köln.

WindHeuser, H. (1977): Die Stellung des Laacher Vulkanismus (Osteifel) im Quartär. - Geologisches Institut der Universität zu Köln, Sonderveröffentlichungen, 31: 1-223; Köln.

Manuskript eingegangen am 28.06.1994 